\title{
Envelope tomography of long-period variable stars st, $^{\star}$
}

\section{Line-doubling frequency among Mira stars}

\author{
R. Alvarez ${ }^{1}$, A. Jorissen ${ }^{1, \star \star \star}$, B. Plez ${ }^{2}$, D. Gillet ${ }^{3}$, A. Fokin ${ }^{4}$, and M. Dedecker ${ }^{1, \dagger}$ \\ 1 Institut d'Astronomie et d'Astrophysique, Université Libre de Bruxelles, CP 226, Boulevard du Triomphe, \\ 1050 Bruxelles, Belgium \\ e-mail: ralvarez, ajorisse, dedecker@astro.ulb.ac.be \\ 2 GRAAL, Université Montpellier II, cc072, 34095 Montpellier Cedex 05, France \\ e-mail: plez@graal.univ-montp2.fr \\ 3 Observatoire de Haute-Provence, 04870 Saint-Michel l'Observatoire, France \\ e-mail: gillet@obs-hp.fr \\ 4 Institute for Astronomy of the Russia Academy of Sciences, 48 Pjatnitskaja, 109017 Moscow, Russia \\ e-mail: fokin@inasan.rssi.ru
}

Received 30 March 2001 / Accepted 22 August 2001

\begin{abstract}
This paper presents statistics of the line-doubling phenomenon in a sample of 81 long-period variable (LPV) stars of various periods, spectral types and brightness ranges. The set of observations consists of 315 highresolution optical spectra collected with the spectrograph ELODIE at the Haute-Provence Observatory, during 27 observing nights at one-month intervals and spanning two years. When correlated with a mask mimicking a K0III spectrum, $54 \%$ of the sample stars clearly showed a double-peaked cross-correlation profile around maximum light, reflecting double absorption lines. Several pieces of evidence are presented that point towards the double absorption lines as being caused by the propagation of a shock wave through the photosphere. The observation of the Balmer lines appearing in emission around maximum light in these stars corroborates the presence of a shock wave. The observed velocity discontinuities, ranging between 10 and $25 \mathrm{~km} \mathrm{~s}^{-1}$, are not correlated with the brightness ranges. A comparison with the center-of-mass (COM) velocity obtained from submm CO lines originating in the circumstellar envelope reveals that the median velocity between the red and blue peaks is blueshifted with respect to the COM velocity, as expected if the shock moves upwards. The LPVs clearly exhibiting line-doubling around maximum light with the K0III mask appear to be the most compact ones, the stellar radius being estimated from their effective temperatures (via the spectral type) and luminosities (via the period-luminosity relationship). It is not entirely clear whether or not this segregation between compact and extended LPVs is an artefact of the use of the K0III mask. Warmer masks (F0V and G2V) applied to the most extended and coolest LPVs yield asymmetric cross-correlation functions which suggest that line doubling is occurring in those stars as well. Although a firm conclusion on this point is hampered by the large correlation noise present in the CCFs of cool LPVs obtained with warm masks, the occurrence of line doubling in those stars is confirmed by the double CO $\Delta v=3$ lines observed around $1.6 \mu \mathrm{m}$ by Hinkle et al. (1984, ApJS, 56, 1). Moreover, the $\mathrm{H} \delta$ line in emission, which is another signature of the presence of shocks, is observed as well in the most extended stars, although with a somewhat narrower profile. This is an indication that the shock is weaker in extended than in compact LPVs, which may also contribute to the difficulty of detecting line doubling in cool, extended LPVs.
\end{abstract}

Key words. stars: AGB and post-AGB - stars: atmospheres - stars: late-type - stars: oscillations - stars: variables: general - shock waves

Send offprint requests to: A. Jorissen,

e-mail: ajorisse@astro.ulb.ac.be

* Based on observations made at Observatoire de Haute Provence, operated by the Centre National de la Recherche Scientifique, France.

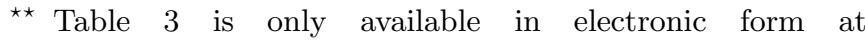
http://www.edpsciences.org and at the CDS via anonymous ftp to cdsarc.u-strasbg.fr $(130.79 .128 .5)$ or via http://cdsweb.u-strasbg.fr/cgi-bin/qcat?J/A+A/379/305

\section{Introduction}

Long-period variable stars (LPVs) are cool giant stars of low and intermediate masses, and the light variations associated with the pulsation of their envelope have long periods (typically $300 \mathrm{~d}$ ) and large amplitudes (up

\footnotetext{
${ }^{\star \star \star}$ Research Associate, F.N.R.S. (Belgium).

$\dagger$ F.R.I.A. Research Assistant (Belgium).
} 
to 9 magnitudes peak-to-peak in the visual). LPVs are subdivided into Mira Ceti-type variables (Mira stars or Miras) and semi-regular variables (of the SRa or SRb subtypes), depending on the light curve amplitude, shape and regularity.

Apart from the brightness fluctuations, LPVs are characterized by striking spectral changes:

- appearance and disappearance of hydrogen and metallic emission lines and splitting of many of the emission lines of the Balmer series into several components (e.g. Merrill 1921, 1923; Merrill \& Burwell 1930; Adams 1941; Joy 1947; Deutsch \& Merrill 1959; Gillet et al. 1983, 1985a, 1985b, 1989; Ferlet \& Gillet 1984; Gillet 1988a, 1988b);

- absorption line shifts correlated with the lower excitation potential of the line (e.g. Adams 1941; Merrill 1947; Fujita 1951; Joy 1954);

- doubling of several absorption lines around maximum light (e.g. Adams 1941; Merrill \& Greenstein 1958; Maehara 1968, 1971; Tsuji 1971; Hinkle 1978; Hinkle \& Barnes 1979a, 1979b; Hinkle et al. 1982, 1984, 1997; Wallerstein 1985; Wallerstein et al. 1985; Barnbaum 1992; Hinkle \& Barnbaum 1996).

Merrill (1955) was the first to suggest that the spectral changes observed in LPVs may be explained by the presence of a shock wave moving outward. This idea has been extensively investigated since then by a number of authors: e.g. Gorbatskii (1957, 1961), Willson (1972, 1976), Hinkle (1978), Willson \& Hill (1979), Hill \& Willson (1979), Wood (1979), Willson et al. (1982), Gillet \& Lafon (1983, 1984, 1990), Fox et al. (1984), Fox \& Wood (1985), Bessell et al. (1996), Fadeyev \& Gillet (2000, 2001).

Nevertheless, due to the lack of a complete and selfconsistent model describing the pulsation and its effect on the spectrum, most of the questions raised by the spectral peculiarities of LPVs remain unanswered so far. For instance, the origin of the emission lines is still debated: although most authors believe they are formed in the hot wake of the shock (Gillet 1988a; de la Reza 1986 and references therein), others reject the shock wave scenario and invoke instead purely non-LTE radiative processes (Magnan \& de Laverny 1997).

The velocity of the shock front is also a matter of debate: large shock-wave velocity discontinuities (of the order of $60 \mathrm{~km} \mathrm{~s}^{-1}$ ) are indeed required to photodissociate $\mathrm{H}_{2}$ molecules and photoionize hydrogen atoms in order to subsequently produce by recombination the observed Balmer emission lines (Gillet et al. 1989). Velocity discontinuities of that order are indeed observed for fluorescent lines (Willson 1976), although double absorption lines yield much lower velocity discontinuities (of the order of $20-30 \mathrm{~km} \mathrm{~s}^{-1}$; e.g. Adams 1941; Merrill \& Greenstein 1958; Maehara 1968; Tsuji 1971; Hinkle 1978; Hill \& Willson 1979; Hinkle et al. 1997 and references therein). The lower velocity discontinuities derived from absorption lines are not necessarily incompatible with the theoretical requirement, since the velocities of the blue and red components suffer from a strong averaging effect due to the large extension of the region where they form.

The line-doubling phenomenon is source of conflicting theories. Some important progress towards its understanding has however been made recently thanks to a dedicated monitoring of Mira variables (Alvarez et al. 2000a, hereafter Paper I), which confirmed that the line-doubling phenomenon is caused by a shock wave propagating in the photosphere. It was shown that the temporal evolution of the red and blue peaks of the double absorption lines of the Mira variable RT Cyg is consistent with the socalled "Schwarzschild scenario". This scenario, originally presented by Schwarzschild (1952) for W Vir Cepheids, relates the evolution of the line profile to the progression of a shock wave in the atmosphere. Alternative models accounting for line doubling without resorting to differential atmospheric motions (Karp 1975; Gillet et al. 1985a) can thus be definitively rejected.

It was shown in Paper I that some LPVs (e.g., X Oph) do not (or, at least, not clearly) exhibit line doubling around maximum light. This paper further investigates the questions raised by this result: what is the frequency of Mira variables exhibiting line-doubling around maximum light (Sect. 4.3.1) and what are their distinctive properties (Sect. 4)? To address these questions, a large sample of LPVs (mostly Mira variables) of various spectral types was monitored during 2 years (Sect. 2).

\section{Sample and observations}

This section describes the two-year-long monitoring of a large sample of LPV stars performed with ELODIE at the Observatoire de Haute-Provence (France) at a frequency of about one night per month, weather-permitting.

\subsection{The spectrovelocimeter ELODIE}

The fibre-fed echelle spectrograph ELODIE (Baranne et al. 1996) is mounted on the $1.93-\mathrm{m}$ telescope of the Observatoire de Haute-Provence (France). This instrument is designed to perform very accurate radialvelocity measurements by cross-correlating the stellar spectrum with numerical masks. In one exposure an echelle spectrum at a resolution of 42000 ranging from $3906 \AA$ to $6811 \AA$ is recorded. Then, an automatic online data processing extracts a 2-D spectrum (67 orders $\times$ 1044 pixels) from the echelle spectrum. We developed our own cross-correlation procedure, following the prescriptions of Baranne et al. (1996). We have thus the possibility of using our own numerical templates and to perform different kinds of tests in the computation of the cross-correlation function $(\mathrm{CCF})$. The $\mathrm{CCF}$ is computed directly from the 2-D spectrum. First, it is wavelengthcalibrated, then corrected from the value of the Earth barycentric velocity towards the star. To preserve the accuracy, the CCF is computed without either rebinning or merging the orders. Only orders having an average signalto-noise $(S / N)$ ratio greater than 2.0 are used. 
Two templates have been systematically used in a first step to compute the CCFs of the whole sample: (i) the default K0 III mask provided in the ELODIE reduction software (Baranne et al. 1996); (ii) a M4 V mask constructed by Delfosse et al. (1999) from an ELODIE spectrum of Barnard's star (Gl 699) applying the method of Baranne et al. (1979). Although the K0III template may in fact seem inadequate to study much cooler LPVs, it was argued in Sect. 2.2 of Alvarez et al. (2001; hereafter Paper II) that "warm" masks like the K0III one are more prone to detect line doubling (see also Sects. 3 and 4.3.2.1). On the other hand, it has been checked a posteriori (see the discussion in Sect. 4.3.2.1 in relation with Fig. 10) that the M4V mask yields CCFs that are identical to those obtained with a mask mimicking the spectrum of the M4.5III star HD 123657 (see Table 1).

\subsection{The sample}

The sample is composed of: (i) 6 circumpolar Miras (SU Cam, S UMi, RT Dra, AX Cep, S Cep, RY Cep) that can be observed all along the pulsational cycle; (ii) 76 LPVs (Mira and semi-regular variables) of different periods, chemical compositions, spectral types and brightness ranges, observed at different phases; (iii) 8 non-LPV red giants; (iv) 3 radial-velocity standard stars. The entire sample (93 stars) is presented in Table 1: for each star are indicated its GCVS (General Catalogue of Variable Stars, Kholopov et al. 1988) name (or the HD number); its right ascension and declination (2000.0); its variability type, period, spectral type and brightness range as given by the GCVS; the number of observations $N$. The last column indicates whether the star is circumpolar ("circum."), nonLPV ("non-LPV") or radial-velocity standard ("r.v. st.").

\subsection{The observations}

A total of 27 observing nights on ELODIE were allocated between 1998, August and 2000, August (more or less one night per month). Approximatively one third of the total observing time was lost mainly because of bad atmospheric conditions, causing interruptions in the phase coverage. About 15-20 stars were observed during each clear night, with typical exposure times of about $25 \mathrm{~min}$ yielding a $S / N$ ratio per resolution element at $500 \mathrm{~nm}$ up to 150 for the brightest stars. For the faintest and reddest stars, sometimes only the reddest orders recorded a usable signal. But the power of a correlation technique is precisely that it does not require high $S / N$ spectra to deliver useful CCFs (see e.g., Queloz 1995). As indicated in Sect. 2.1, the CCF is computed using orders having an average $S / N$ ratio of at least 2.0 .

The $\log$ of observations is presented in Table 2: the first and second columns list the civil date and the corresponding Julian date at midnight. The third column gives the code number of the night: each observing night will be subsequently referred to by this number. Only the nights during which at least one star was observed are reported in Table 2.

A total of 315 spectra were collected, i.e. 3-4 spectra per star on average. Some (circumpolar) stars were observed as often as 12 times (S Cep), and the majority of variable stars were observed $2-3$ times.

\section{The variety of cross-correlation profiles and their frequency of occurrence}

For each of the 315 spectra, we compute the CCFs with the K0 III and the M4 V default templates. A large variety of CCFs are observed among LPVs, ranging from the classical single-peak profile to much more complex profiles (asymmetrical peak, double peak, "noisy" profiles, etc.). We developed an automatic classification procedure which classifies the CCFs according to their shape and contrast into a finite number of archetypes, thus avoiding any subjectivity. Table $3^{1}$ provides for each observation the CCF code for the K0- and M4-templates, together with the night number, the Julian date, the phase and, when appropriate, the heliocentric radial velocities as derived by a single or double gaussian fit of the CCF. The different kinds of CCFs observed are as follows:

- Single peak: the most common profile observed is the classical single peak (Fig. 1a). In this case, the minimum of the CCF is supposedly the radial velocity of the star, at least for the non-variable stars. For variable stars, the observed velocity might be perturbed by atmospheric motions and may thus not correspond to the center-of-mass velocity (it is worth noting that these intrinsic radial-velocity variations render the search for binaries among LPVs extremely difficult; see Sect. 4.4). Single-peak profiles occur at all phases. $54 \%$ of the LPV sample stars showed at least once this kind of profile with the K0-template ( $28 \%$ of the total number of observations). These values amount respectively to $89 \%$ and $74 \%$ with the M4-template. This cool template thus yields single-peak, well-contrasted CCFs much more often than the K0-template. These profiles are coded " $1 \mathrm{p}$ " in Table 3 ;

- Double peak and asymmetrical peak: the crosscorrelation technique permits to very clearly reveal the doubling of the lines despite the severe crowding of the LPV spectra (Fig. 1b). Sometimes, the profile only exhibits an asymmetry, as if the double peak was about to appear (or to disappear) (Fig. 1c).

A double or asymmetrical peak was observed for $50 \%$ of the LPV stars (28\% of the total number of observations) with the K0-template. Most of them were not known in the literature to exhibit the line-doubling phenomenon. The doubling is essentially observed

1 Only available in the electronic version of this paper; see also http://www-astro.ulb.ac.be/tomography.html 
Table 1. Sample stars.

\begin{tabular}{|c|c|c|c|c|c|c|c|c|}
\hline Name & $\begin{array}{l}\text { Right Asc. } \\
(2000.0)\end{array}$ & $\begin{array}{l}\text { Declination } \\
(2000.0)\end{array}$ & $\begin{array}{l}\text { Variability } \\
\text { Type }\end{array}$ & $\begin{array}{l}\text { Period } \\
\text { (d) }\end{array}$ & $\begin{array}{l}\text { Spectral } \\
\text { Type }\end{array}$ & $\begin{array}{r}\text { Brightness } \\
\text { Range }\end{array}$ & $N$ & Comments \\
\hline SV And & 00:04:20 & $+40: 06: 36$ & Mira & 316.21 & M5e-M7e & $7.7-14.3$ & 2 & \\
\hline $\mathrm{T}$ And & $00: 22: 23$ & $+26: 59: 46$ & Mira & 280.76 & $\mathrm{M} 4 \mathrm{e}-\mathrm{M} 7.5 \mathrm{e}$ & $7.7-14.5$ & 2 & \\
\hline R And & $00: 24: 02$ & $+38: 34: 39$ & Mira & 409.33 & $\mathrm{~S} 3,5 \mathrm{e}-\mathrm{S} 8,8 \mathrm{e}$ & $5.8-14.9$ & 1 & \\
\hline Y Cep & $00: 38: 22$ & $+80: 21: 24$ & Mira & 332.57 & M5e-M8.2e & $8.1-16.0$ & 1 & \\
\hline $\mathrm{U}$ Psc & $01: 22: 58$ & $+12: 41: 54$ & Mira & 173.10 & $\mathrm{M} 4 \mathrm{e}$ & $10.3-14.9$ & 1 & \\
\hline R Psc & $01: 30: 38$ & $+02: 52: 55$ & Mira & 344.50 & M3e-M6e & $7.0-14.8$ & 5 & \\
\hline Y And & $01: 39: 37$ & $+39: 20: 37$ & Mira & 220.53 & M3e-M4.5e & $8.2-15.1$ & 5 & \\
\hline$o$ Cet & $02: 19: 21$ & $-02: 58: 28$ & Mira & 331.96 & M5e-M9e & $2.0-10.1$ & 3 & \\
\hline R Cet & $02: 26: 02$ & $-00: 10: 42$ & Mira & 166.24 & M4e-M9 & $7.2-14.0$ & 7 & \\
\hline $\mathrm{U}$ Cet & $02: 33: 44$ & $-13: 08: 54$ & Mira & 234.76 & M2e-M6e & $6.8-13.4$ & 1 & \\
\hline R Tri & 02:37:02 & $+34: 15: 54$ & Mira & 266.9 & M4IIIe-M8e & $5.4-12.6$ & 3 & \\
\hline U Ari & $03: 11: 03$ & $+14: 47: 58$ & Mira & 371.13 & M4e-M9.5e & $7.2-15.2$ & 5 & \\
\hline SS Cep & $03: 49: 30$ & $+80: 19: 20$ & $\mathrm{SRb}$ & 90.0 & M5III & $8.0-9.1$ & 3 & \\
\hline $\mathrm{R}$ Tau & $04: 28: 18$ & $+10: 09: 44$ & Mira & 320.90 & M5e-M9e & $7.6-15.8$ & 4 & \\
\hline V Tau & $04: 52: 02$ & $+17: 32: 18$ & Mira & 168.7 & M0e-M4.5e & $8.5-14.6$ & 4 & \\
\hline R Aur & $05: 17: 18$ & $+53: 35: 11$ & Mira & 457.51 & M6.5e-M9.5e & $6.7-13.9$ & 3 & \\
\hline W Aur & $05: 26: 55$ & $+36: 54: 11$ & Mira & 274.27 & M3e-M8e & $8.0-15.3$ & 2 & \\
\hline RU Aur & $05: 40: 08$ & $+37: 38: 12$ & Mira & 466.47 & M7e-M9e & $9.0-16.0$ & 3 & \\
\hline S Cam & $05: 41: 02$ & $+68: 47: 55$ & $\mathrm{SRa}$ & 327.26 & $\mathrm{C} 7,3 \mathrm{e}(\mathrm{R} 8 \mathrm{e})$ & $7.7-11.6$ & 5 & \\
\hline$\alpha$ Ori & $05: 55: 10$ & $+07: 24: 25$ & SRc & & M1 & 0.50 & 1 & non-LPV \\
\hline U Ori & $05: 55: 49$ & $+20: 10: 31$ & Mira & 368.3 & M6e-M9.5e & $4.8-13.0$ & 4 & \\
\hline$\delta$ Aur & $05: 59: 31$ & $+54: 17: 11$ & & & Ko & 3.71 & 1 & non-LPV \\
\hline X Aur & $06: 12: 13$ & $+50: 13: 41$ & Mira & 162.79 & M3e-M7e & $8.0-13.6$ & 2 & \\
\hline$\mu \mathrm{Gem}$ & $06: 22: 57$ & $+22: 30: 54$ & $\mathrm{Lb}$ & & M3 & 2.97 & 1 & non-LPV \\
\hline SU Cam & $06: 38: 12$ & $+73: 55: 00$ & Mira & 285.03 & M5 & $8.9-12.6$ & 8 & circum. \\
\hline X Gem & $06: 47: 07$ & $+30: 16: 35$ & Mira & 264.16 & M5e-M8e(Tc:) & $7.5-13.8$ & 3 & \\
\hline X Mon & $06: 57: 12$ & $-09: 03: 51$ & SRa & 155.80 & M1eIII-M6ep & $6.8-10.2$ & 1 & \\
\hline R Gem & $07: 07: 21$ & $+22: 42: 13$ & Mira & 369.91 & $\mathrm{~S} 2.9 \mathrm{e}-\mathrm{S} 8.9 \mathrm{e}$ & $6.0-14.0$ & 7 & \\
\hline $\mathrm{R}$ CMi & $07: 08: 43$ & $+10: 01: 27$ & Mira & 337.78 & $\mathrm{C} 7,1 \mathrm{Je}(\mathrm{CSep})$ & $7.25-11.6$ & 1 & \\
\hline $\mathrm{S} \mathrm{CMi}$ & $07: 32: 43$ & $+08: 19: 07$ & Mira & 332.94 & M6e-M8e & $6.6-13.2$ & 5 & \\
\hline$v$ Gem & $07: 35: 55$ & $+26: 53: 50$ & & & M0 & 4.06 & 1 & non-LPV \\
\hline 81 Gem & $07: 46: 08$ & $+18: 30: 39$ & & & $\mathrm{~K} 4$ & 4.87 & 1 & non-LPV \\
\hline $\mathrm{R}$ Cnc & $08: 16: 34$ & $+11: 43: 35$ & Mira & 361.6 & M6e-M9e & $6.07-11.8$ & 5 & \\
\hline X UMa & $08: 40: 49$ & $+50: 08: 11$ & Mira & 249.04 & M3e-M4e & $8.1-14.8$ & 4 & \\
\hline HD 76830 & $08: 59: 11$ & +18:08:09 & & & M4 & 6.38 & 1 & non-LPV \\
\hline UZ Hya & $09: 16: 45$ & $-04: 36: 24$ & Mira & 260.95 & $\mathrm{M} 4 \mathrm{e}$ & $8.8-14.5$ & 5 & \\
\hline R Leo & $09: 47: 33$ & $+11: 25: 46$ & Mira & 309.95 & M6e-M8IIIe & $4.4-11.3$ & 3 & \\
\hline $\mathrm{S} \mathrm{LMi}$ & $09: 53: 43$ & $+34: 55: 32$ & Mira & 233.83 & $\mathrm{M} 2.0 \mathrm{e}-\mathrm{M} 8.2 \mathrm{e}$ & $7.5-14.3$ & 2 & \\
\hline V Leo & 10:00:02 & $+21: 15: 40$ & Mira & 273.35 & M5e & $8.4-14.6$ & 4 & \\
\hline R UMa & $10: 44: 39$ & $+68: 46: 33$ & Mira & 301.62 & M3e-M9e & $6.5-13.7$ & 4 & \\
\hline RU UMa & $11: 41: 40$ & $+38: 28: 30$ & Mira & 252.46 & M3e-M5e & $8.1-15.0$ & 4 & \\
\hline Y Vir & $12: 33: 52$ & $-04: 25: 18$ & Mira & 218.43 & M2e-M5e & $8.3-15.0$ & 4 & \\
\hline R Vir & $12: 38: 30$ & $+06: 59: 18$ & Mira & 145.63 & M3.5IIIe-M8 & $6.1-12.1$ & 2 & \\
\hline RS UMa & $12: 38: 57$ & $+58: 29: 03$ & Mira & 258.97 & M4e-M6e & $8.3-14.9$ & 4 & \\
\hline S UMa & $12: 43: 57$ & $+61: 05: 36$ & Mira & 225.87 & S0,9e-S5,9e & $7.1-12.7$ & 2 & \\
\hline U Vir & $12: 51: 06$ & $+05: 33: 12$ & Mira & 206.64 & M2e-M8e: & $7.4-13.5$ & 2 & \\
\hline V UMi & $13: 38: 41$ & $+74: 18: 37$ & SRb & 72.0 & M5IIIab & $7.2-9.1$ & 5 & \\
\hline SY Vir & $\begin{array}{l}13: 58: 38 \\
13: 51\end{array}$ & $-04: 34: 35$ & Mira & 236.65 & M6: & $9.6-13.4$ & 1 & \\
\hline HD 123657 & $14: 07: 56$ & $+43: 51: 18$ & $\mathrm{Lb}$ & & M4.5III & 5.25 & 1 & non-LPV \\
\hline$\alpha$ Boo & $14: 15: 38$ & $+19: 11: 06$ & & & K1.5III & -0.04 & 1 & r.v. st \\
\hline R Boo & $14: 37: 12$ & $+26: 44: 12$ & Mira & 223.4 & M3e-M8e & $6.2-13.1$ & 5 & \\
\hline Y Lib & $15: 11: 41$ & $-06: 00: 43$ & Mira & 275.70 & $\mathrm{M} 5 \mathrm{e}-\mathrm{M} 8.2 \mathrm{e}$ & $7.6-14.7$ & 4 & \\
\hline RT Boo & $15: 17: 15$ & $+36: 21: 34$ & Mira & 273.86 & M6.5e-M8e & $8.3-13.9$ & 2 & \\
\hline S Ser & $15: 21: 40$ & $+14: 18: 52$ & Mira & 371.84 & M5e-M6e & $7.0-14.1$ & 1 & \\
\hline S UMi & $15: 29: 35$ & $+78: 38: 00$ & Mira & 331.0 & M6e-M9e & $7.5-13.2$ & 10 & circum. \\
\hline ST Her & $15: 50: 47$ & $+48: 29: 00$ & $\mathrm{SRb}$ & 148.0 & M6 & $8.8-10.3$ & 1 & \\
\hline RU Her & $16: 10: 15$ & $+25: 04: 14$ & Mira & 484.83 & M6e-M9 & $6.8-14.3$ & 7 & \\
\hline SS Oph & $16: 57: 52$ & $-02: 45: 42$ & Mira & 180.64 & M5e & $7.8-14.5$ & 1 & \\
\hline RV Her & $17: 00: 35$ & $+31: 13: 22$ & Mira & 205.23 & M2e & $9.0-15.5$ & 1 & \\
\hline SY Her & $17: 01: 29$ & $+22: 28: 40$ & Mira & 116.91 & M1e-M6e & $8.4-14.0$ & 1 & \\
\hline $\mathrm{Z} \mathrm{Oph}$ & $17: 19: 32$ & $+01: 30: 52$ & Mira & 348.7 & К3ер-M7.5 & $7.6-14.0$ & 10 & \\
\hline RS Her & $17: 21: 42$ & $+22: 55: 16$ & Mira & 219.70 & M4e-M8 & $7.0-13.0$ & 3 & \\
\hline $\mathrm{RU} \mathrm{Oph}$ & $17: 32: 53$ & $+09: 25: 24$ & Mira & 202.29 & M3e-M5e & $8.6-14.2$ & 1 & \\
\hline$\beta \mathrm{Oph}$ & $17: 43: 27$ & $+04: 34: 03$ & & & K2III & 2.77 & 6 & r.v. st \\
\hline T Her & 18:09:06 & $+31: 01: 16$ & Mira & 164.98 & M2,5e-M8e & $6.8-13.7$ & 1 & \\
\hline RY Oph & $18: 16: 37$ & $+03: 41: 34$ & Mira & 150.41 & M3e-M6 & $7.4-13.8$ & 1 & \\
\hline RT Dra & $18: 19: 26$ & $+72: 40: 50$ & Mira & 279.41 & M5 & $9.6-13.8$ & 14 & circum. \\
\hline SV Her & $18: 26: 23$ & $+25: 01: 35$ & Mira & 238.99 & M5e & $9.1-15.1$ & $\begin{array}{r}14 \\
1\end{array}$ & \\
\hline $\mathrm{X} O \mathrm{ph}$ & $18: 38: 21$ & $+08: 50: 01$ & Mira & 328.85 & M5e-M9e & $5.9-9.2$ & 4 & \\
\hline WZ Lyr & $19: 02: 15$ & $+47: 12: 56$ & Mira & 376.64 & M9e & $10.6-15.0$ & 2 & \\
\hline RU Lyr & $19: 12: 21$ & $+41: 18: 12$ & Mira & 371.84 & M6e:-M8e & $9.5-15.9$ & 1 & \\
\hline W Aql & $19: 15: 23$ & $-07: 02: 50$ & Mira & 490.43 & $\mathrm{~S} 3,9 \mathrm{e}-\mathrm{S} 6,9 \mathrm{e}$ & $7.3-14.3$ & 1 & \\
\hline RT Cyg & $19: 43: 38$ & $+48: 46: 41$ & Mira & 190.28 & M2e-M8.8eIb & $6.0-13.1$ & 7 & \\
\hline$\gamma \mathrm{Aql}$ & $19: 46: 14$ & $+10: 36: 43$ & & & K3II & 2.72 & 3 & r.v. st \\
\hline$\chi \mathrm{Cyg}$ & $19: 50: 34$ & $+32: 54: 53$ & Mira & 408.05 & $\mathrm{~S} 6,2 \mathrm{e}-\mathrm{S} 10,4 \mathrm{e}$ & $3.3-14.2$ & 6 & \\
\hline
\end{tabular}


Table 1. continued.

\begin{tabular}{llllllrll}
\hline Name & $\begin{array}{l}\text { Right Asc. } \\
(2000.0)\end{array}$ & $\begin{array}{l}\text { Declination } \\
(2000.0)\end{array}$ & $\begin{array}{l}\text { Variability } \\
\text { Type }\end{array}$ & $\begin{array}{l}\text { Period } \\
(\mathrm{d})\end{array}$ & $\begin{array}{l}\text { Spectral } \\
\text { Type }\end{array}$ & $\begin{array}{r}\text { Brightness } \\
\text { Range }\end{array}$ & $N$ & Comments \\
\hline Z Cyg & $20: 01: 27$ & $+50: 02: 34$ & Mira & 263.69 & M5e-M9e & $7.1-14.7$ & 1 & \\
S Aql & $20: 11: 37$ & $+15: 37: 13$ & SRa & 146.45 & M3e-M5.5e & $8.9-12.8$ & 1 & \\
Z Aql & $20: 15: 11$ & $-06: 09: 04$ & Mira & 129.22 & M3e & $8.2-14.8$ & 1 & \\
WX Cyg & $20: 18: 34$ & $+37: 26: 54$ & Mira & 410.45 & C8,2JLi(N3e) & $8.8-13.2$ & 2 & \\
T Cep & $21: 09: 32$ & $+68: 29: 28$ & Mira & 388.14 & M5.5e-M8.8e & $5.2-11.3$ & 6 & \\
RR Aqr & $21: 15: 01$ & $-02: 53: 43$ & Mira & 182.45 & M2e-M4e & $9.1-14.4$ & 1 & \\
X Peg & $21: 21: 00$ & $+14: 27: 00$ & Mira & 201.20 & M2e-M5e & $8.8-14.4$ & 1 & \\
SW Peg & $21: 22: 29$ & $+21: 59: 45$ & Mira & 396.33 & M4e & $8.0-14.0$ & 2 & \multirow{2}{*}{ circum. } \\
AX Cep & $21: 26: 52$ & $+70: 13: 18$ & Mira & 395.0 & C(N) & $9.5-13.0$ & 11 & circum. \\
S Cep & $21: 35: 13$ & $+78: 37: 28$ & Mira & 486.84 & C7,4e(N8e) & $7.4-12.9$ & 12 & circer \\
RV Peg & $22: 25: 38$ & $+30: 28: 22$ & Mira & 396.80 & M6e & $9.0-15.5$ & 1 & \\
AR Cep & $22: 51: 33$ & $+85: 02: 47$ & SRb & & M4III & $7.0-7.9$ & 2 & non-LPV \\
R Peg & $23: 06: 39$ & $+10: 32: 35$ & Mira & 378.1 & M6e-M9e & $6.9-13.8$ & 4 & \\
W Peg & $23: 19: 50$ & $+26: 16: 44$ & Mira & 345.5 & M6e-M8e & $7.6-13.0$ & 5 & \\
S Peg & $23: 20: 33$ & $+08: 55: 08$ & Mira & 319.22 & M5e-M8.5e & $6.9-13.8$ & 3 & \\
RY Cep & $23: 21: 14$ & $+78: 57: 31$ & Mira & 149.06 & Ke-M0e & $8.6-13.6$ & 9 & circum. \\
ST And & $23: 38: 45$ & $+35: 46: 17$ & SRa & 328.34 & C4,3e-C6,4e & $7.7-11.8$ & 5 & \\
R Cas & $23: 58: 24$ & $+51: 23: 18$ & Mira & 430.46 & M6e-M10e & $4.7-13.5$ & 4 & \\
\hline
\end{tabular}

around maximum light (between phases -0.1 and 0.3 ), as can be seen in Fig. 2. With the M4-template, only $23 \%$ of sample stars ( $10 \%$ of the total number of observations) showed these kinds of profiles (see Sect. 2.2 of Paper II for a discussion of the physical origin of this difference between the K0- and the M4-templates), and the phases range from -0.2 to 0.6 . These profiles are coded "2p" (double peak) or "ap" (asymmetrical peak) in Table 3;

- Doubtful single/double peak and noisy profile: the profiles cannot always be unambiguously classified as single or double peak, because of the poor contrast and/or the complex shape of the CCFs. Such profiles can only be classified as "doubtful single profiles" or "doubtful double profiles" (Figs. 1d and 1e, coded 1p? and 2p?, respectively, in Table 3 ). In some cases, the contrast of the CCF is so low that the cross-correlation profile does not even yield any clear dip; no radial velocity can be derived from such noisy CCFs (Fig. 1f), identified as "noisy profiles" (coded "np" in Table 3). The distinction between "doubtful single/double-peak profile" and "noisy profile" is somewhat arbitrary (compare Figs. 1e and 1f) and depends upon the particular (and somewhat arbitrary) values adopted for the parameters involved in our automatic classification procedure. No attempt to derive radial velocities was made with these kinds of profiles.

It must be noted that the general structure of the CCFs presented in Figs. 1d and 1e is in fact strikingly similar, the only difference being the respective contrasts of the leftmost and second-to-leftmost peaks. Moreover, a given star (like R Cet; see Table 3 and Fig. 1) may evolve from $1 p$ ? CCFs to $2 p$ ? CCFs and even to $2 \mathrm{p}$ at different phases of its light cycle, thus
Table 2. Log of observations.

\begin{tabular}{lll}
\hline $\begin{array}{l}\text { Date of } \\
\text { Observations }\end{array}$ & $\begin{array}{l}\text { Julian Date } \\
(2450000+)\end{array}$ & $\begin{array}{l}\text { Night } \\
\text { Number }\end{array}$ \\
\hline 1998 Aug., 05-06 & 1031.5 & N1 \\
1998 Sep., 03-04 & 1060.5 & N2 \\
1998 Sep., 28-29 & 1085.5 & N3 \\
1998 Oct., 06-07 & 1093.5 & N4 \\
1998 Dec., 23-24 & 1171.5 & N5 \\
1999 Jan., 26-27 & 1205.5 & N6 \\
1999 Feb., 23-24 & 1233.5 & N7 \\
1999 Apr., 22-23 & 1291.5 & N9 \\
1999 May, 19-20 & 1318.5 & N10 \\
1999 Jul., 05-06 & 1365.5 & N12 \\
1999 Jul., 26-27 & 1386.5 & N13 \\
1999 Sep., 02-03 & 1424.5 & N14 \\
1999 Sep., 28-29 & 1450.5 & N15 \\
1999 Nov., 16-17 & 1499.5 & N16 \\
1999 Dec., 16-17 & 1529.5 & N17 \\
2000 Jan., 10-11 & 1554.5 & N18 \\
2000 Feb., 22-23 & 1597.5 & N19 \\
2000 Feb., 23-24 & 1598.5 & N20 \\
2000 Apr., 17-18 & 1652.5 & N22 \\
2000 May, 18-19 & 1683.5 & N23 \\
2000 Jun., 20-21 & 1716.5 & N24 \\
2000 Jul., 11-12 & 1737.5 & N25 \\
2000 Aug., 09-10 & 1766.5 & N26 \\
2000 Aug., 10-11 & 1767.5 & N27 \\
\hline
\end{tabular}

suggesting that the $1 \mathrm{p}$ ? and $2 \mathrm{p}$ ? CCFs of the kind displayed in Figs. 1d and 1e may be intrinsically similar and should in fact be classified together (that conclusion may in fact even be extended to some "np" profiles, like the one displayed in Fig. 1f whose general 


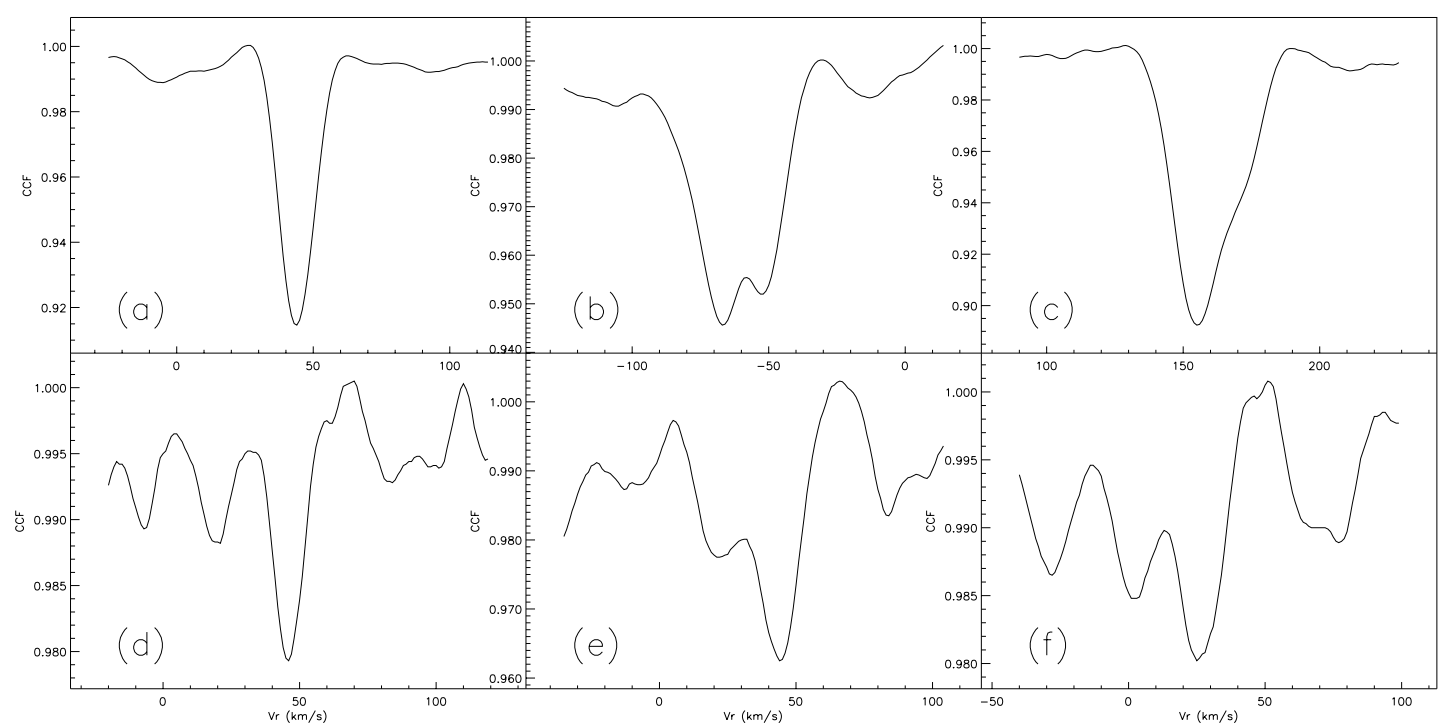

Fig. 1. Examples of the different kinds of CCFs observed: a) single peak: R Cet, night N7, phase 0.96, mask M4V; b) double peak: R Boo, night N7, phase 0.98, mask K0III; c) asymmetrical peak: X Mon, night N22, phase 0.94, mask K0III; d) doubtful single peak: R Cet, night N18, phase 0.89, mask K0III; e) doubtful double peak: R Cet, night N4, phase 0.30, mask K0III; f) noisy profile: R Cnc, night N3, phase 0.96, mask K0III.

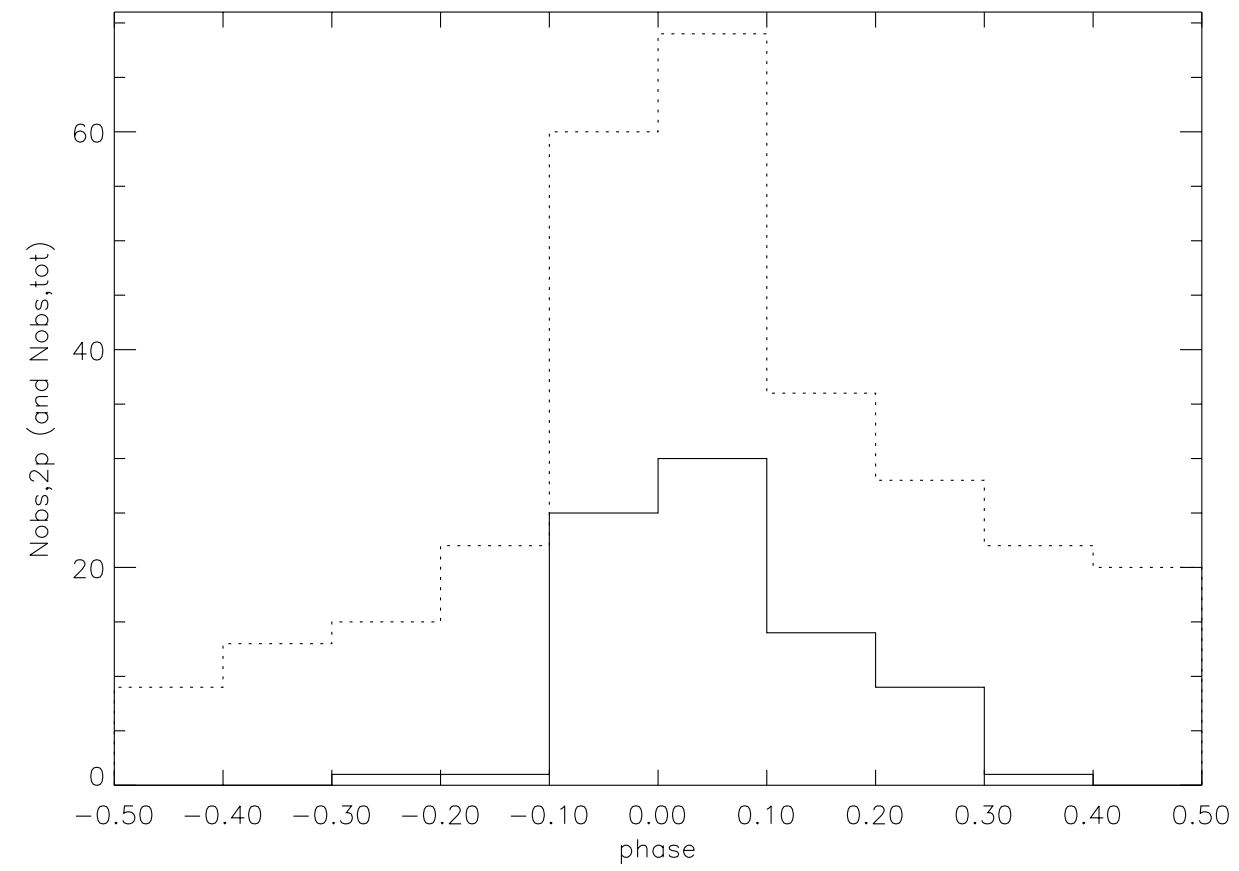

Fig. 2. Phase distributions (i) for the observations which exhibit a double or an asymmetrical peak with the K0-template (solid line) and (ii) for the total set of observations (dashed line).

structure resembles that of Figs. 1d and 1e). However, in this first analysis of the statistics of the line-doubling phenomenon, it was decided to adopt conservative classification criteria, at the risk of excluding physicallysound data. In particular, the line-doubling statistics presented in Figs. 2, 6-9 relies only on CCFs classified as "2p" or "ap" and excludes CCFs classified as "2p?" and "np". The possible physical implications of this conservative choice are discussed further in Sect. 4.3.1.1.
Doubtful or noisy profiles were obtained at least once for $61 \%$ of the LPV sample stars (44\% of the total number of observations) with the K0-template. Although these profiles were found at all phases, they are preferentially observed around minimum light (when the star is fainter and cooler). With the M4-template, the above percentages turn to only $17 \%$ of the stars (most of which are the S-type and C-type stars of the sample) and $16 \%$ of the total number of observations. 
Figure 2 presents the distribution in phase of the doublepeak profiles as compared to the total number of observations. The striking features exhibited by Fig. 2 are (i) the very sharp rise in the fraction of double-peak profiles at phase -0.1 , (ii) the total absence of double-peak profiles between phases 0.4 and 0.7 , and (iii) the fraction of double-peak profiles remains almost constant $(\sim 40 \%)$ between phases -0.1 and 0.3 .

Another conclusion that can be drawn at this point concerns the interest of the cross-correlation technique for dynamical studies: as already pointed out in Paper I, the cross-correlation technique provides a powerful tool to extract double lines despite the severe crowding of the spectra of giant stars (see however the discussion of Sect. 4.3.2.1 relative to late-type LPVs). The study of the spectral variations associated with the pulsation of LPV stars has no more to be restricted to the few clean nearIR spectral lines. The much richer visible spectrum is now accessible as well to these studies, opening great potentialities as illustrated for instance by the tomographic technique described in Paper II.

\section{The Schwarzschild scenario}

The origin of the line-doubling phenomenon in LPVs has been a long-standing problem (see Paper I for a review). The most definitive way to prove that the line doubling is not the result of purely radiative processes but is actually caused by the differential bulk motions in the atmosphere arising from the propagation of a shock wave is to check whether or not the temporal sequence of the line doubling follows the Schwarzschild scenario.

Schwarzschild (1952) developed his scenario for W Vir variables, but in principle it can be generalized to any variable star where a shock wave propagates through the photosphere. According to this scenario, the temporal evolution of the intensity of the components of a double line should obey a precise sequence: the intensity of the blue component, formed in deeper, ascending photospheric layers, should increase from zero to maximum, while at the same time the red component, formed in the upper infalling photospheric layers, decreases from maximum to zero (Fig. 1 of Paper I).

Thanks to a two-month-long monitoring of two LPVs around maximum light, it was shown in Paper I that (at least some) LPVs do follow the Schwarzschild scenario. The present set of observations of many LPVs spread over two years enables us to answer the following questions in relation with the line-doubling phenomenon: (i) at which phases does it occur? (ii) does it repeat identically from one cycle to the next? (iii) what is the fraction of LPV stars exhibiting the line doubling phenomenon and what are their distinctive properties?

\subsection{Temporal evolution of the line doubling}

Although it was shown in Paper I that the line profile of the Mira RT Cyg followed the Schwarzschild scenario around maximum light, there was no information available about the evolution of the profile at later phases.

In the present sample, the circumpolar Mira SCep (C7, 4e; $P=486.84 \mathrm{~d}$ according to the GCVS) has a good phase coverage and allows to draw the velocity curve over a full light cycle (Fig. 3): a typical S-shaped curve emerges that is very similar to the curves obtained from the rotation-vibration $\mathrm{CO}$ or $\mathrm{CN}$ lines in the near-infrared (Hinkle et al. 1997 and references therein). S Cep was already known to exhibit the line-doubling phenomenon for those lines (Hinkle \& Barnbaum 1996; Fig. 3).

Figure 4 presents the sequence of CCFs obtained for S Cep. Several important features must be noted: (i) as seen in Fig. 3, the velocities of the red and blue components are almost identical from one cycle to the next (although their relative intensities change somewhat: compare the profiles at phases 0.95 and 1.96 on Fig. 4); (ii) the center-of-mass velocity derived from the submm CO lines falls in between the red and blue peaks, as expected (Figs. 3 and 4; see also Sect. 4.2); (iii) the velocities derived from the near infrared CO lines (Hinkle et al. 1997) are very similar to those derived from the optical CCFs.

\subsection{Line doubling and center-of-mass velocity}

The location of the center-of-mass (COM) velocity with respect to the blue and red peaks provides important information about the shock. In the classical Schwarzschild scenario, the COM velocity falls in between the blue and red peaks. It might happen, however, that the shock is not strong enough to oppose the infalling matter. In that case, both peaks would be redshifted with respect to the COM velocity (indicating that the shock is receding in a Eulerian rest frame), as observed for RR Lyr stars at some phases (Chadid \& Gillet 1996).

This does not occur in S Cep, as seen in Figs. 3 and 4 (see also Hinkle \& Barnbaum 1996) where the dotted line corresponds to the COM velocity derived by Neri et al. (1998) from sub-mm observations. And seemingly, receding shocks are not observed in Miras at all. Table 4 lists the COM velocity (when available) for LPVs which exhibit a double-peak CCF. The first column gives the star name, the second column the velocities of the blue and red components (extreme value observed), the third column the median of the blue- and red-peak velocities, the fourth column the COM velocity derived from sub-mm observations and the last column the reference for this COM velocity. The COM velocity does always fall in between the blue and red peaks (the median of the red- and blue-peak velocities is in fact blueshifted with respect to the COM velocity, as expected if the shock front is moving outwards), in agreement with the conclusion already reached by Hinkle et al. (1997) from infrared CO lines. It is thus possible to conclude that, unlike in other classes of variable stars such as RR Lyrae, shock waves are rather strong in Mira stars. 


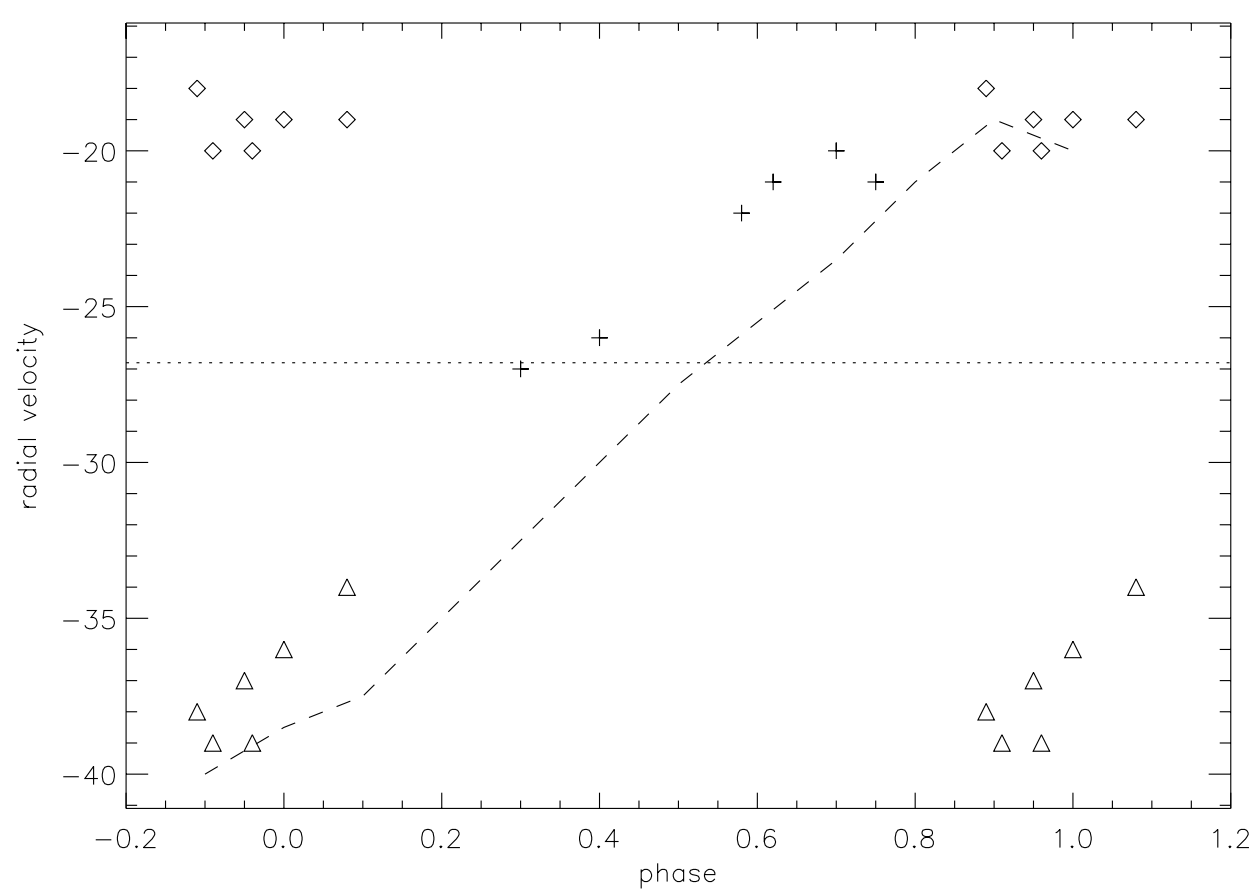

Fig. 3. Velocity variations of S Cep (cross: single component; triangles: blue component; diamonds: red component). The dotted line indicates the center-of-mass velocity (see Table 4). The velocities between phases 0.8 and 1.1 are shown twice to illustrate the velocity behaviour through an entire cycle. The dashed line is an eye fit to the radial-velocity curve of $\mathrm{S}$ Cep obtained by Hinkle \& Barnbaum (1996) from CN $\Delta v=-2$ red-system lines around $2 \mu \mathrm{m}$ (their Fig. 1).

Table 4. Center-of-mass velocity for stars exhibiting a doublepeak $\mathrm{CCF}$.

\begin{tabular}{rcccc}
\hline GCVS & $\begin{array}{c}\text { CCF } \\
\text { radial velocities } \\
\left(\mathrm{km} \mathrm{s}^{-1}\right)\end{array}$ & $\begin{array}{c}\text { median } \\
\text { red/blue } \\
\left(\mathrm{km} \mathrm{s}^{-1}\right)\end{array}$ & $\begin{array}{c}\text { Center-of-mass } \\
\text { velocity } \\
\left(\mathrm{km} \mathrm{s}^{-1}\right)\end{array}$ & Ref. \\
\hline R And & $-38 /-10$ & -24 & -20.3 & 2 \\
R Cet & $+22 /+48$ & +35 & +42.1 & 1 \\
U Ari & $-65 /-43$ & -54 & -46.5 & 1 \\
R Gem & $-57 /-39$ & -48 & -47.6 & 2 \\
S CMi & $+50 /+72$ & +61 & +65.9 & 2 \\
W Aql & $-54 /-27$ & -40.5 & -39.2 & 2 \\
Z Cyg & $-179 /-160$ & -169.5 & -165.8 & 1 \\
AX Cep & $-10 /+8$ & -1 & +0.4 & 1 \\
S Cep & $-39 /-18$ & -28.5 & -26.8 & 3 \\
\hline
\end{tabular}

References:

1: Groenewegen et al. (1999).

2: Knapp et al. (1998).

3: Neri et al. (1998).

\subsection{The key stellar parameter governing the occurrence of the Schwarzschild scenario}

\subsubsection{Statistics of the line-doubling phenomenon}

Not all LPVs exhibit the line doubling (at least when considering only those CCFs classified as "2p" or "ap" as described in Sect. 3, thus excluding CCFs of the "2p?" and "np" kinds; see Fig. 1), even when observed at maximum light (see the example of X Oph in Paper I): about $47 \%$ of the sample stars observed at maximum light (between phases -0.10 and 0.10 ) did not exhibit line doubling with the K0-template (see Fig. 2). In an attempt to identify the key stellar parameter(s) governing the appearance of the line doubling, the distribution of several potentially relevant parameters is displayed in Fig. $5\left(V_{\text {red }}-V_{\text {blue }}\right.$ against the brightness range), Fig. 6 (period distribution) and Fig. 7 (distribution of spectral type at maximum light for oxygen-rich LPVs).

There is a weak correlation between the brightness range and the velocity discontinuity (Fig. 5), but the scatter in the relation is very large, albeit similar to that obtained by Hinkle et al. (1997; their Fig. 11) when considering only Miras. The important result from Fig. 5 is that there is no visual-amplitude threshold beyond which line doubling only would occur: instead, small-amplitude as well as large-amplitude Miras are liable to exhibit the line-doubling phenomenon.

The same conclusion is reached when considering the period (Fig. 6) or spectral type (Fig. 7) distributions: double-peak profiles are found among short- as well as long-period LPVs and among early- as well as late-type LPVs. The frequency of double-peak profiles is nevertheless somewhat larger among short-period and early-type LPVs (almost all LPVs with a period less than $250 \mathrm{~d}$ and almost all oxygen-rich LPVs with a spectral type earlier than M3 showed line-doubling), suggesting that the linedoubling is restricted to LPVs of small radius (Sect. 4.3.2). 

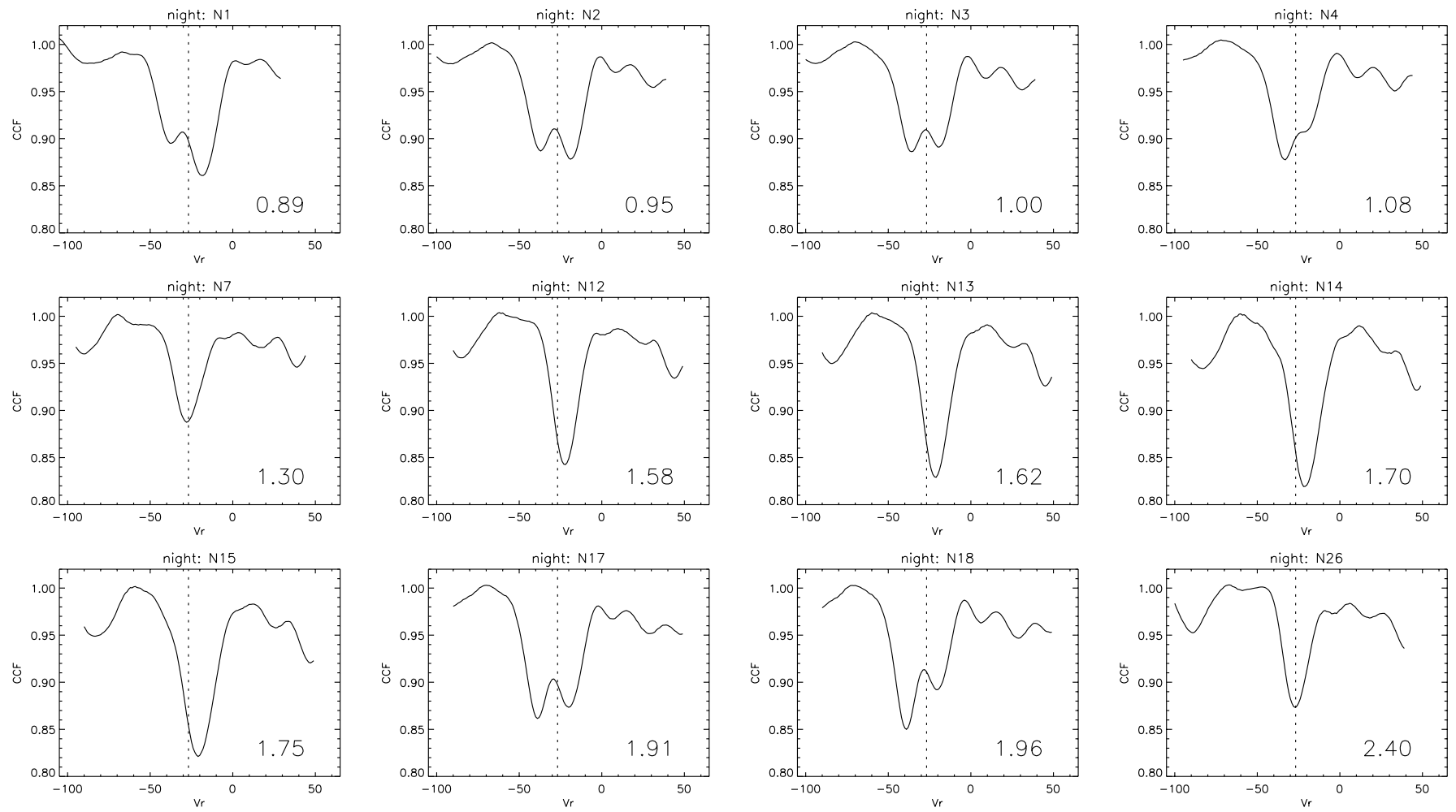

Fig. 4. Sequence of CCFs for S Cep with the default K0 III mask. The labels beside each CCF refer to the visual phase computed from the GCVS period and the AAVSO ephemeris. The observing night number is given on top of each panel according to Table 2. The dotted line indicates the center-of-mass velocity (see Table 4).

\subsubsection{The stellar radius: The key parameter?}

Since the period is correlated with the luminosity and the spectral type with the effective temperature, and since these two parameters suffice to define a stellar radius, we investigate the relationship between the line-doubling phenomenon and the quantity $\rho / R_{\odot}=\left[\left(L / L_{\odot}\right)\left(T / T_{\odot}\right)^{-4}\right]^{1 / 2}$. Although this quantity is certainly related to the stellar radius, it should not be taken at face value since the definition of a stellar radius for LPVs is a complex task (e.g. Perrin et al. 1999; Hofmann et al. 2000). The quantity $\rho$ should somehow reflect the stellar size, at least in a relative sense when comparing one star to another.

Luminosities were estimated from the $M_{\mathrm{bol}}-P$ relation of Feast et al. (1989), with no zero-point correction.

Effective temperatures of LPVs are also difficult to derive. Apart from a scarce number of direct determinations from interferometric observations, little is known about the temperature scale of Mira and semi-regular variables. Colour-temperature relations may in principle be used (e.g. Bessell et al. 1998; Alvarez et al. 2000b; Houdashelt et al. 2000), but in practice it is difficult to collect the required set of simultaneous photometric observations for a large sample of LPVs.

In order to estimate $T_{\text {eff }}$ for a number of sample stars as large as possible, the choice was made to rely on a spectral type-temperature relation, taking advantage of the fact that almost all LPVs have been given a spectral type, at least at maximum light. The spectral type/temperature scale of Perrin et al. (1998) was used for this purpose, with the spectral types at maximum light taken from the GCVS. As spectral-type assignments generally carry some degree of subjectivity, so will the $T_{\text {eff }}$ values derived from the present procedure. Their accuracy will be sufficient for the present purpose of identifying a possible relationship between $\rho$ and the line-doubling phenomenon. The derived $\rho$ values are listed in Table 3 .

Figure 8 shows the $\rho$ distribution for two sub-samples: (i) the stars for which a double or asymmetrical peak was obtained with the K0-template (solid line); (ii) the stars for which a double or asymmetrical peak was not obtained, even when observed at maximum light (dashed line). Semi-regular variables (as they do not follow the period-luminosity relation) and carbon stars (as they do not obey the oxygen-rich spectral type/temperature relation) are excluded from Fig. 8. Stars with a double peak are clearly separated from single-peak stars. A large majority of small-size stars $\left(\rho<200 R_{\odot}\right)$ present a double peak, whereas large-size stars $\left(\rho>200 R_{\odot}\right)$ are almost all single-peak stars. Such a segregation is quite remarkable given the various sources of uncertainty on $\rho$. It accounts for the results of Paper I, namely the occurrence of line-doubling in RT Cyg $\left(\rho=130 R_{\odot}\right)$ but not in X Oph $\left(\rho=216 R_{\odot}\right)$.

The predictive power of Fig. 8 is of interest in relation with abundance analyses of LPVs in external systems, where the available spectral resolution may not be high 


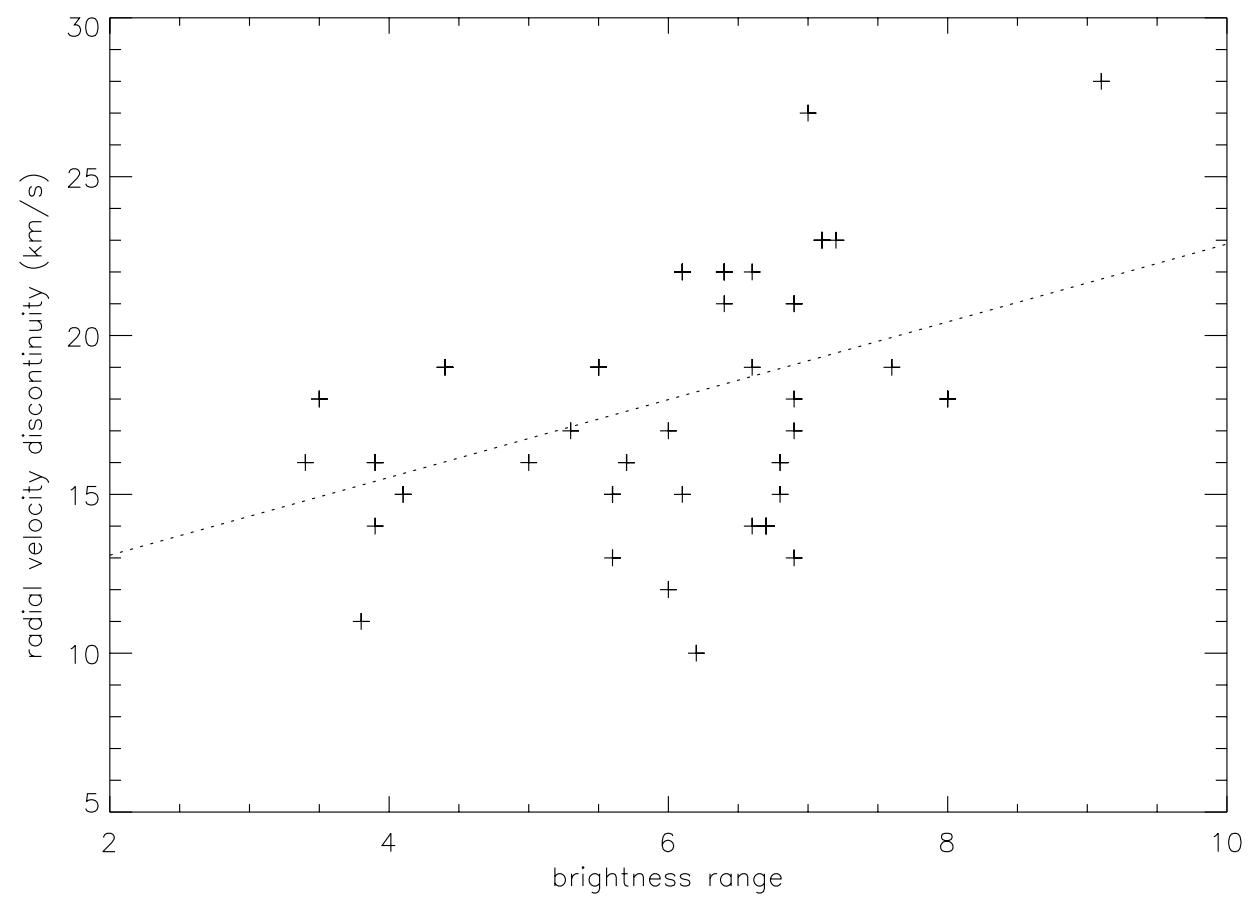

Fig. 5. Radial velocity discontinuity $\left(V_{\text {red }}-V_{\text {blue }}\right)$ against the brightness range (expressed in mag) for stars with a double or asymmetrical peak when observed with the K0-template in the phase range $-0.1-0.1$. The velocity discontinuity displayed corresponds to the maximum value recorded for the above phase range. The dashed line is the regression line.

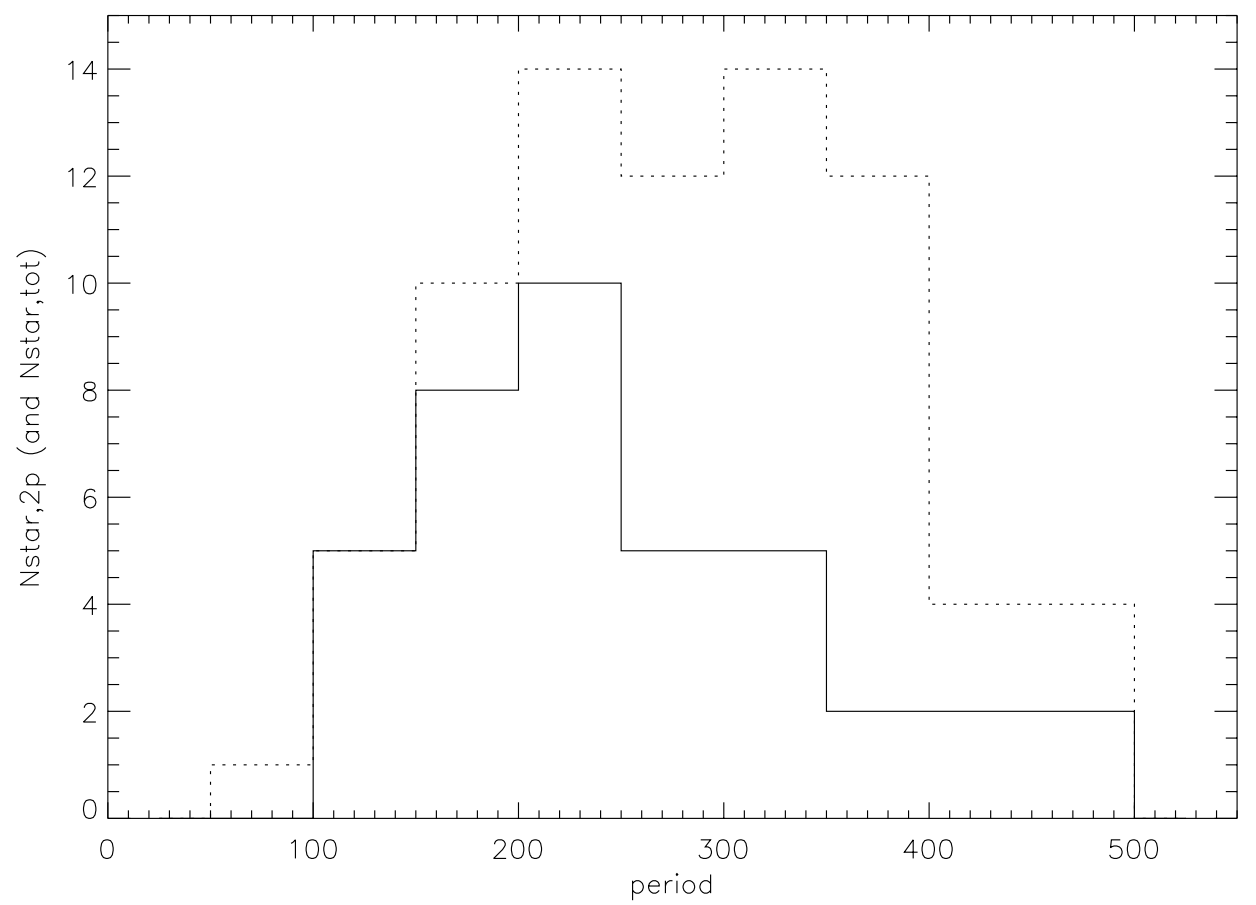

Fig. 6. Period distribution for stars with a double or asymmetrical peak obtained with the K0-template (solid line) as compared to the sample of stars observed at least once around maximum light (dashed line).

enough to reveal the line doubling. This may introduce large errors on the abundances, and Fig. 8 reveals that such a risk is highest for warm and short-period LPVs.

Figure 8, which shows that the line-doubling phenomenon is only present in the most compact Mira stars, is one of the most striking result of this paper. At this stage, two interpretations of this result are still possible: (i) it is an artefact of the use of the KOIII template which is not able to reveal the double peaks in the LPVs with large $\rho$ which have very late and crowded spectra; (ii) the observed segregation in terms of $\rho$ is real and related to the physics of the shocks in LPVs. 


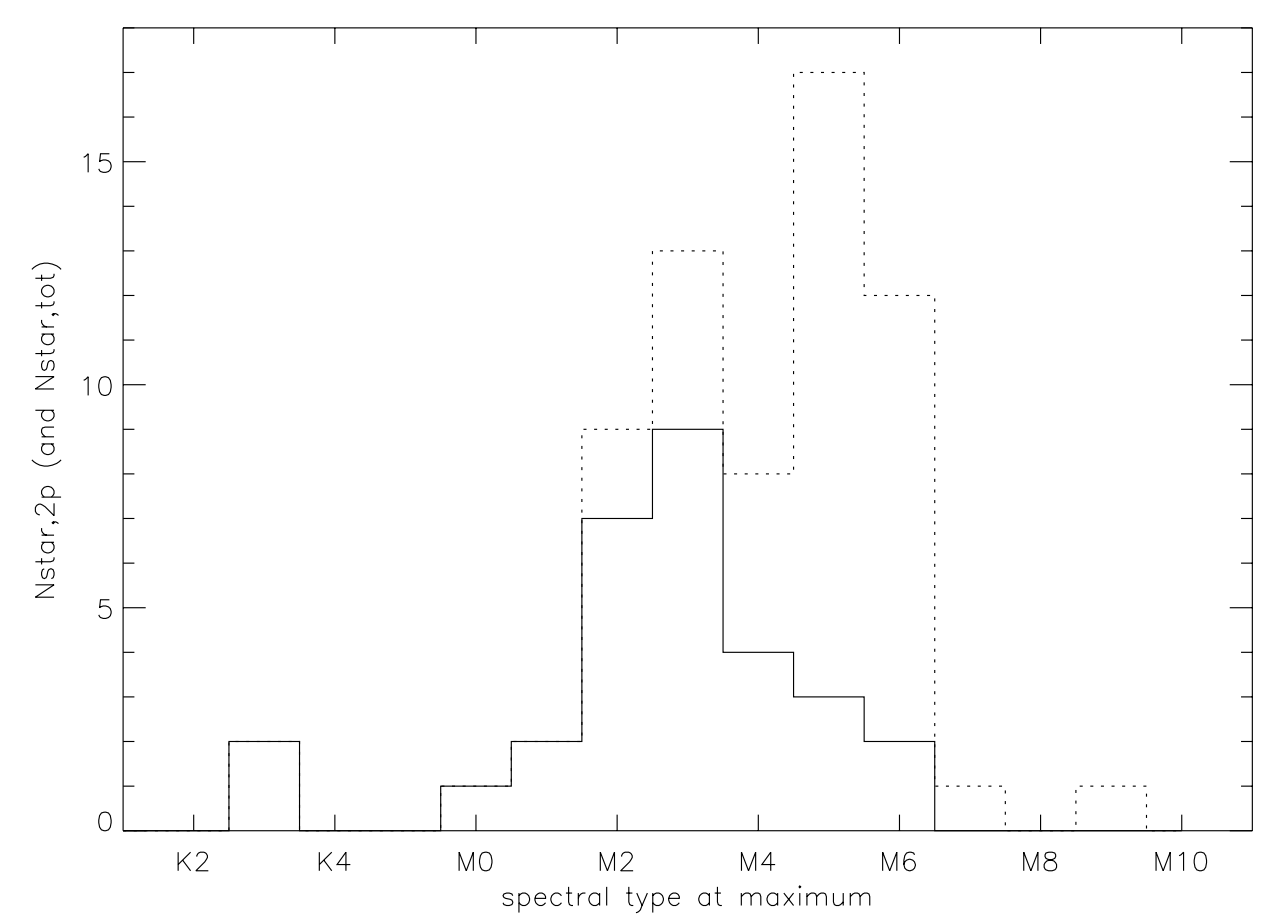

Fig. 7. Distribution of spectral types (at maximum light) for oxygen-rich stars with a double or asymmetrical peak obtained with the K0-template (solid line) as compared to the sample of stars observed at least once around maximum light (dashed line).

These two possibilities are discussed in turn in the remainder of this section.

\subsubsection{An artefact of the method?}

Since the K0III template better matches the spectra of early-type LPVs than of late-type LPVs, it may possibly be more efficient in detecting line-doubling in early-type LPVs, thus possibly causing a bias against the detection of line doubling in large- $\rho$ LPVs. The interpretation of Fig. 8 simply in terms of such a selection bias caused by the use of too warm a template appears however too simplistic, as we now discuss.

Since the cooler M4V template ${ }^{2}$ constitutes a better match to the crowded spectra of late-type LPVs, one might have expected a better efficiency of detection of line doubling with that template. We already showed in Sect. 3 that even less double-peaked CCFs are found with the M4V template than with the K0III one. Figure 9 shows that the segregation of single/double peaks in terms of $\rho$ remains basically the same with the M4V template as with the K0III template.

Nevertheless, by considering infrared CO $\Delta v=3$ lines around $1.6 \mu \mathrm{m}$, Hinkle et al. $(1984,1997)$ reported line doubling around maximum light for several late-type LPVs with large $\rho$ (Table 5) that were flagged as $1 \mathrm{p}$ or $1 \mathrm{p}$ ? with the K0III and M4V templates. Thus line doubling is definitely present at least in some of those stars as well.

\footnotetext{
${ }^{2}$ We show below - see Fig. 10 - that the use of a M4III template does not improve the situation, since it provides CCFs that are very similar to those obtained with the M4V template.
}

Quite interestingly, Hinkle et al. (1984) note that in these late-type LPVs line doubling is more easily seen in the deep photospheric layers probed by the infrared CO $\Delta v=3$ lines around $1.6 \mu \mathrm{m}$ than in the higher layers where the blue-violet absorption lines form. In an attempt to uncover double-peak CCFs in late-type LPVs as well, their spectra were correlated with various masks (constructed with the procedure of Baranne et al. 1979) mimicking F0V, G2V (solar), K4III (81 Gem) and M4III (HD 123657) spectra.

While the cool masks (M4V and M4III) yield very clean, albeit single, CCFs, interesting results are indeed obtained with the warm F0V and G2V masks, which are expected to probe deeper layers. They reveal asymmetric, or even double-peak, CCFs (Table 5 and Fig. 10), with velocities similar to those obtained by Hinkle et al. (1984). There is however a drawback to the application of warm templates to cool LPVs. Since the warm templates contain far fewer lines than the spectrum of cool LPVs, many spurious secondary peaks must be expected in their CCFs. These peaks correspond to the chance coincidence occurring between the mask and the forest of lines flanking the lines actually probed by the mask. This situation is well illustrated by Fig. 10 which compares the CCFs obtained with different masks applied on the late-type LPV R Cas and on the synthetic spectrum of a static $2800 \mathrm{~K}$ model. The latter CCFs make it possible to identify the spurious secondary peaks caused by the correlation noise invoked above. By comparison, the CCFs of R Cas obtained with the warm templates exhibit the same spurious peaks as the $2800 \mathrm{~K}$ model, except that a supplementary blue 


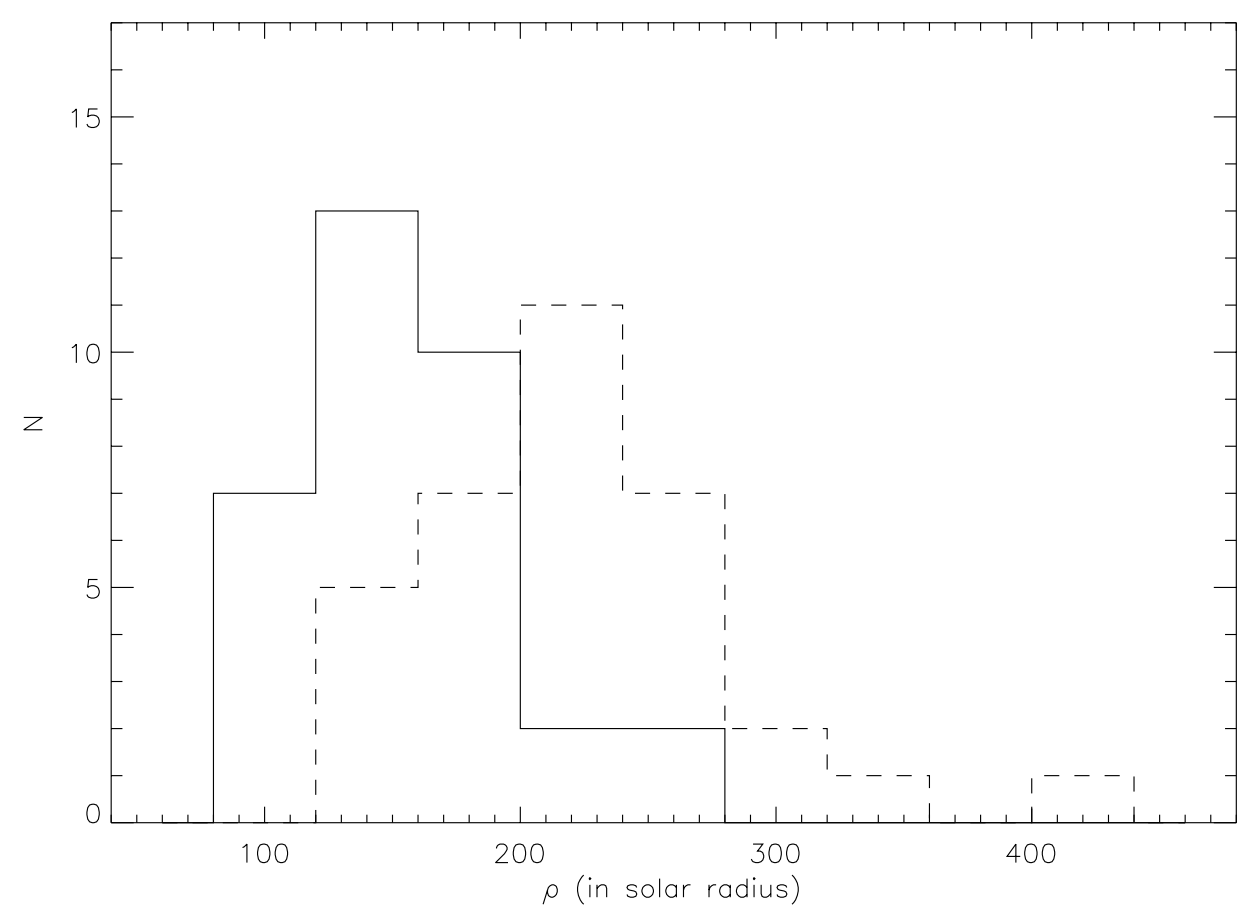

Fig. 8. Distribution of the stellar size $\rho$ (see text) for stars with a double or asymmetrical peak obtained with the K0-template (full line) and for stars for which a double or asymmetrical peak has not been obtained even when observed at maximum light (dashed line).

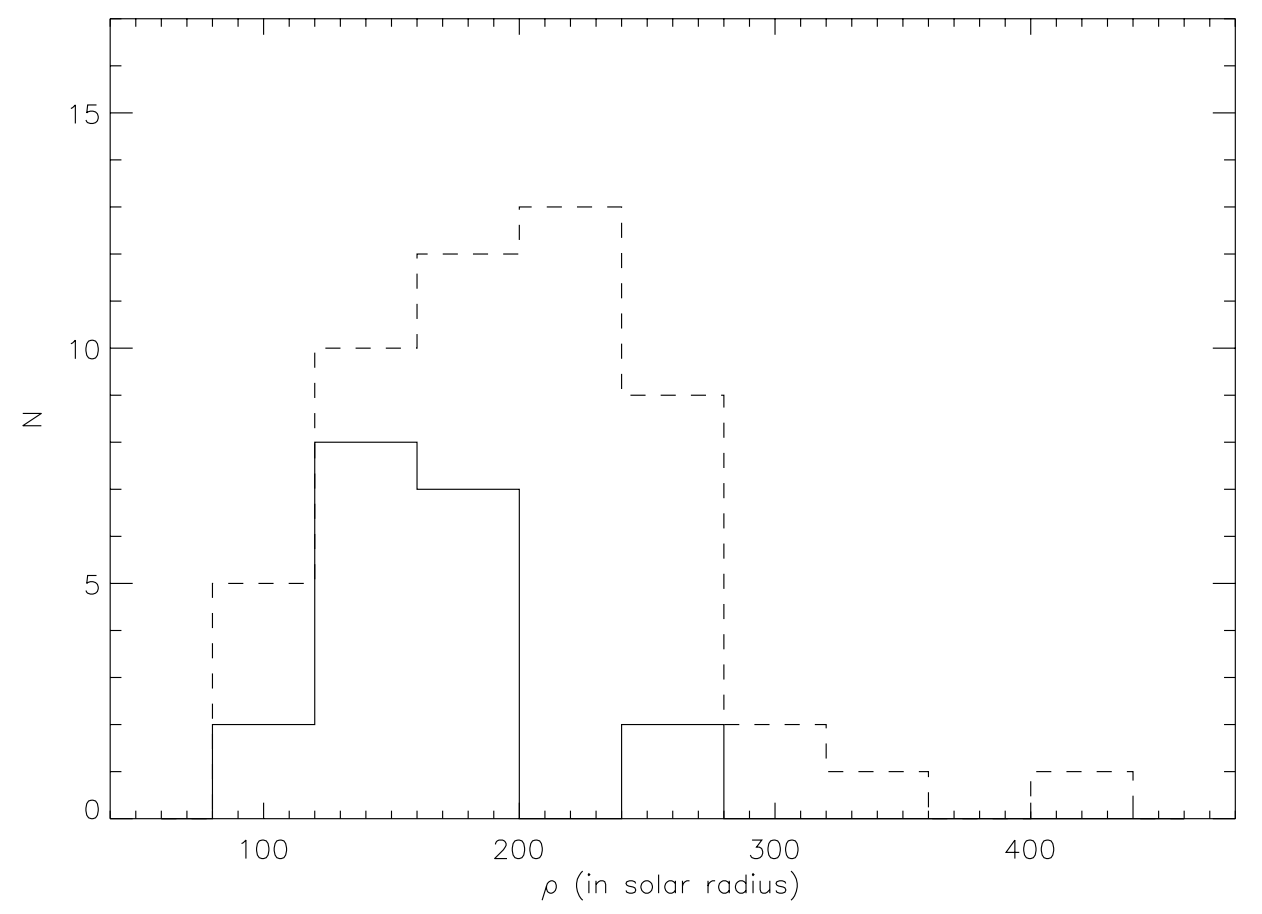

Fig. 9. Same as Fig. 8 for the M4-template.

component appears around $0 \mathrm{~km} \mathrm{~s}^{-1}$ (see also Table 5). This component not observed in the CCF of a static spectrum may thus tentatively be related to the dynamics of the envelope of R Cas.

Many of the LPVs with large $\rho$ that were originally classified as $1 \mathrm{p}$ or $1 \mathrm{p}$ ? from their K0III CCF actually behave like R Cas as shown in Fig. 10.
In conclusion, double-peaked CCFs may actually be present in LPVs with large $\rho$ as well. We nevertheless stress that this conclusion must be subject to caution, since these double-peak CCFs - if real - are quite difficult to detect because of the crowded nature of their parent spectra. In Sect. 4.3.2.2, we discuss the possibility that this difficult detection may also be caused partly by the 
Table 5. Late-type LPVs for which line doubling has been observed by Hinkle et al. (1984) using CO $\Delta v=3$ lines around $1.6 \mu \mathrm{m}$ : comparison of the infrared velocities with those derived from various CCFs at about the same phase.

\begin{tabular}{|c|c|c|c|c|c|c|c|c|c|c|c|c|}
\hline & & & \multicolumn{2}{|c|}{$\mathrm{CO} \Delta v=3$} & \multicolumn{2}{|c|}{$\mathrm{CCF}$} & \multicolumn{2}{|r|}{ K0III } & \multicolumn{2}{|c|}{ M4V } & \multicolumn{2}{|r|}{ F0V } \\
\hline & Spect. type & $\rho\left(R_{\odot}\right)$ & Phase & $V_{\mathrm{r}}$ & Phase & night & & $V_{\mathrm{r}}$ & & $V_{\mathrm{r}}$ & & $V_{\mathrm{r}}$ \\
\hline $\mathrm{R}$ And & $\mathrm{S} 3,5 \mathrm{e}-\mathrm{S} 8,8 \mathrm{e}$ & 217 & 0.96 & $-29.3 /-5.7$ & 0.98 & N26 & $2 \mathrm{p}$ & $-38.3 /-10.2$ & $1 p$ & -9.5 & $2 \mathrm{p}$ & $-38.1 /-9.8$ \\
\hline R Cas & M6e - M10e & 282 & 0.03 & $+5.9 /+28.5$ & 1.02 & N13 & $1 \mathrm{p} ?$ & - & $1 \mathrm{p}$ & +22.7 & $2 \mathrm{p} ?$ & $0 /+21.7$ \\
\hline Т Cep & M5.5e - M8.8e & 238 & 0.87 & $-19.2 /-2.4$ & 1.91 & N23 & np & - & $1 \mathrm{p}$ & -14.9 & $1 \mathrm{pa}$ & $-19.5 /-10.3$ \\
\hline o Cet & M5e - M9e & 150 & 0.96 & $+44.7 /+67.8$ & 1.91 & N15 & $1 \mathrm{p} ?$ & - & $1 \mathrm{p}$ & +61.7 & $2 \mathrm{p} ?$ & $+38.4 /+57.9$ \\
\hline
\end{tabular}
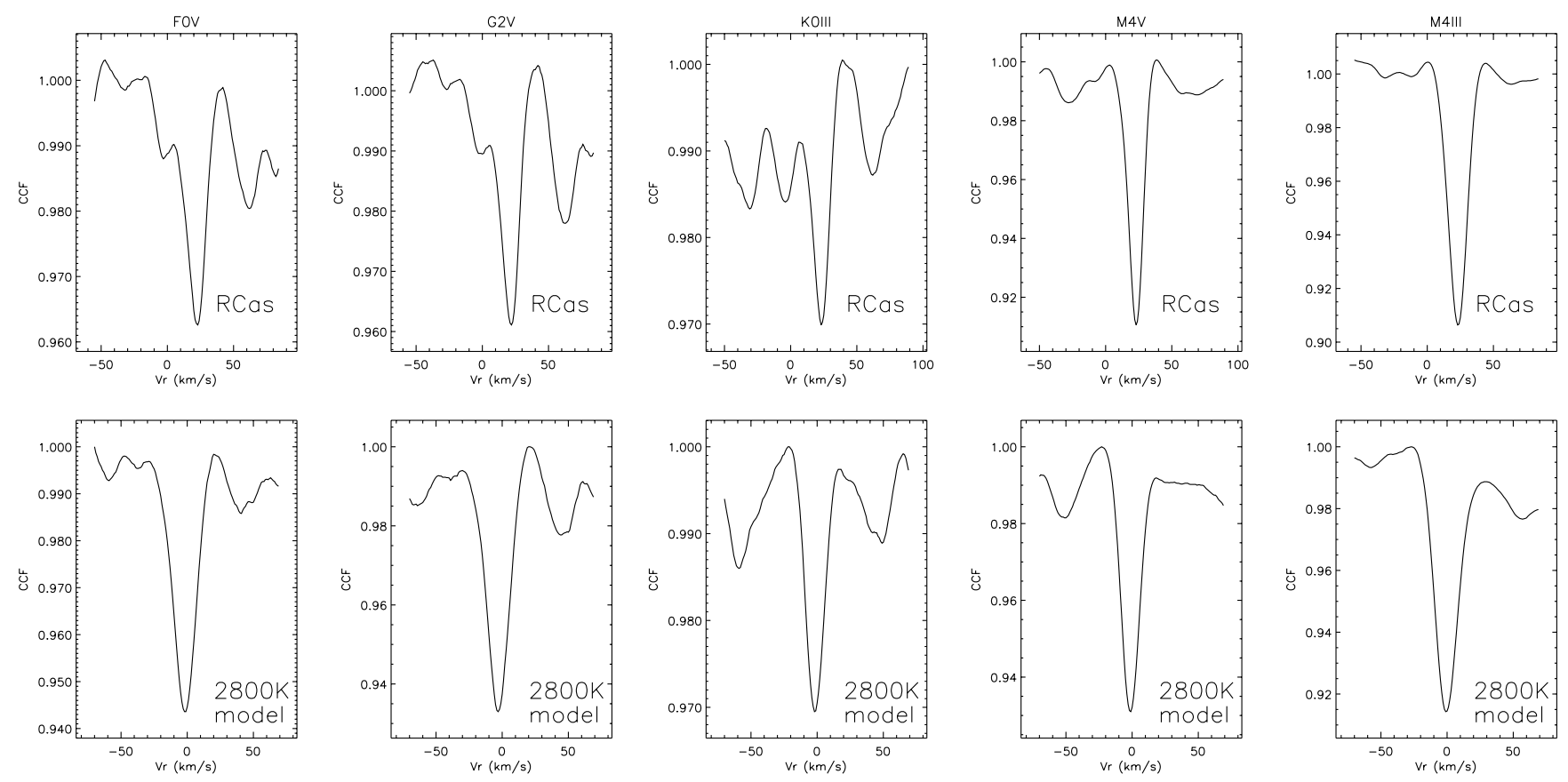

Fig. 10. Upper row: CCFs of R Cas (N13, phase 0.02), a late-type LPV (M6e-M10e), obtained with various templates (F0V, solar G2V, K0III, M4V and M4III). Lower row: CCFs obtained with the same templates applied on a $2800 \mathrm{~K}$ synthetic spectrum (see Sect. 3.1 of Paper II). Note how the CCFs of R Cas are strikingly similar (especially their far wings) to those of the synthetic spectrum at $2800 \mathrm{~K}$, with the exception of the secondary blue peak observed at about $0 \mathrm{~km} \mathrm{~s}^{-1}$ in the F0V, G2V and K0III CCFs of R Cas. This peak might thus tentatively be attributed to the dynamics of the envelope of R Cas, since a corresponding peak is not present in the CCF of a static model atmosphere.

secondary peaks being truly weaker in stars with large $\rho$ due to weaker shocks.

\subsubsection{Weaker shocks in stars with large $\rho$ ?}

The behaviour of the $\mathrm{H} \delta$ emission line in our sample of LPVs provides important clues to help us interpret, in terms of the properties of the shocks in LPVs, the result that the line-doubling phenomenon is conspicuous only in the most compact LPVs.

The first important result regarding $\mathrm{H} \delta$ is that it appears in emission around maximum light for all LPVs in our sample (see Fig. 11 discussed below). Since $\mathrm{H} \delta$ is considered as a good signature of the presence of shocks in those atmospheres, the above result thus suggests that double-peak CCFs, being another signature of shocks, should be observed as well in all LPVs. We argue in the present section that the behaviour of $\mathrm{H} \delta$ and the statistics of double-peak CCFs in LPVs may be reconciled by realizing that the shock strength becomes smaller in stars of larger $\rho$. Figure 11 presents the full width at half maximum (FWHM) of the $\mathrm{H} \delta$ emission line vs. the stellar size $\rho$. We observe a trend of decreasing $\mathrm{H} \delta$ FWHMs with increasing stellar sizes $\rho$. Among the Balmer lines, $\mathrm{H} \delta$ is the best indicator of the energy transferred by the shock to the gas, since $\mathrm{H} \delta$ suffers the least from mutilations induced by molecular absorptions (the TiO line absorption and $\mathrm{H}_{2}$ scattering coefficients are indeed minimum around $\mathrm{H} \delta$; Gillet 1988b). Its width measures the temperature in the post-shock gas where the Balmer emission lines form. The heating in the shock wake must in turn be related to the energy carried by the shock. 


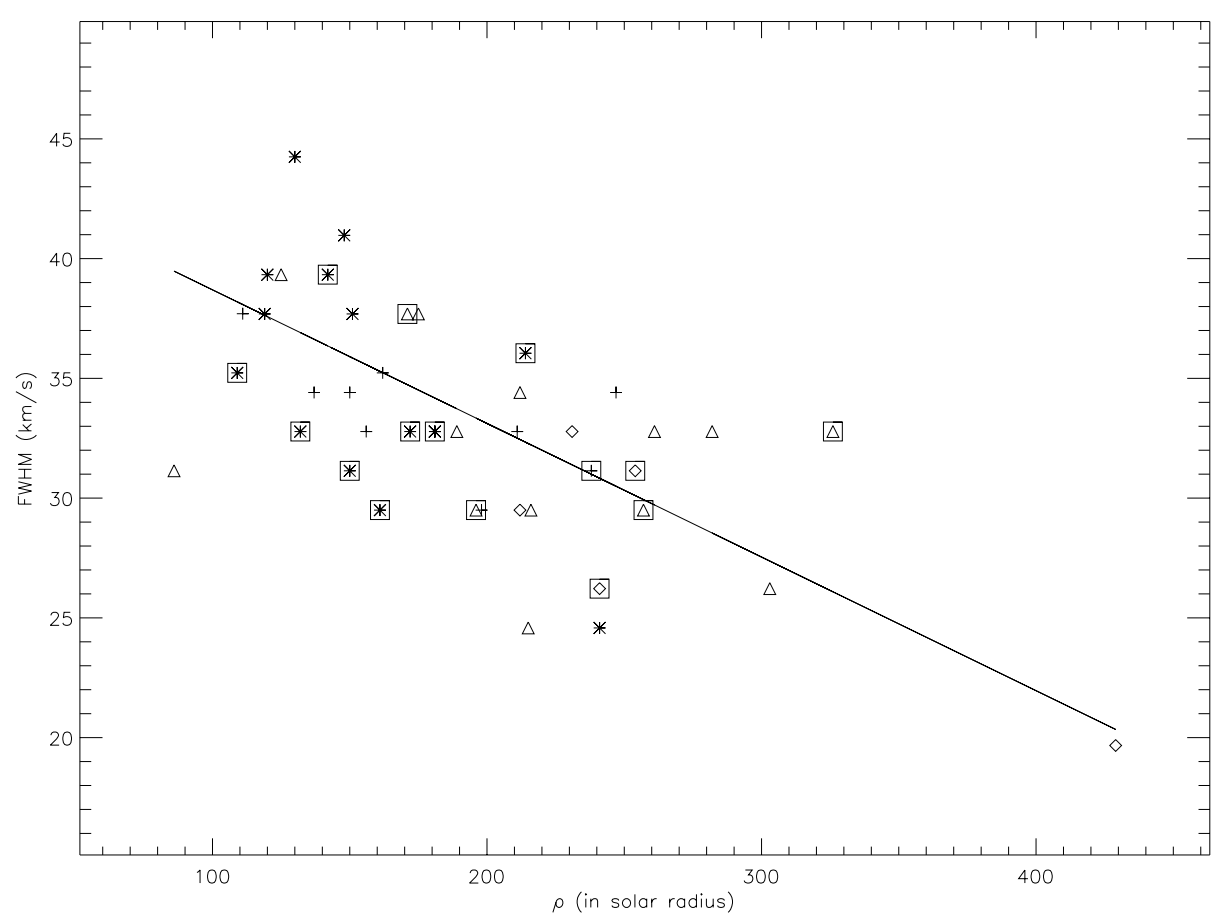

Fig. 11. Full width at half maximum of the $\mathrm{H} \delta$ emission line as a function of the stellar size $\rho$. The solid line is a least-square fit through the data. The different symbols refer to the different types of CCFs obtained with the K0III template as defined in Table 3: asterisks $=2 \mathrm{p}$, crosses $=\mathrm{ap}$, triangles $=1 \mathrm{p}$, diamonds $=\mathrm{np}$. A large square around the previous symbols denotes stars with a double $\mathrm{H} \delta$ profile. The FWHM for these profiles has been measured under the assumption that the self-reversal model applies to them. This model supposes that the emission profile is mutilated by a true absorption line caused by hydrogen located above the shock in the radiative precursor (Gillet 1988b). The "true" FWHM is that corresponding to the non-mutilated emission line. It has been estimated along the guidelines (involving Gaussian fits) described in Fig. 5 of Gillet (1988b). The accuracy of such measurements is estimated to be about $10 \%$.

Similarly, the difficulty of detecting double-peaked CCFs in stars with large $\rho$ might also somehow relate to the weakness of the shocks in those stars (as inferred from their smaller $\mathrm{H} \delta \mathrm{FWHMs}$ ), although a definite proof of this statement would require solving of the equation of radiative transfer in dynamical atmospheres, with detailed shock models.

Self-consistent dynamical models of LPV atmospheres are clearly necessary to confirm that the strongest shocks only occur in the more compact Mira stars. In any case, the relation of the shock strength with the stellar size empirically revealed by Fig. 11 will have to be accounted for by future dynamical models of LPV atmospheres.

\subsection{Repeatability of the line doubling from one cycle to the other and search for binaries}

Observations spread over several cycles are available for some stars and allow us to address the questions of (i) the repeatability of the line doubling from one cycle to the other (as far as the relative peak intensities and velocities are concerned), and (ii) the frequency of spectroscopic binaries among LPVs. In Figs. 12 and 13 are presented the CCFs obtained for R Gem and ST And respectively, at maximum light for two consecutive pulsational cycles. The CCF profile is remarkably similar from one cycle to the other, with the same velocities to within $1 \mathrm{~km} \mathrm{~s}^{-1}$ for the blue and red peaks. This result, based on only two stars, may not necessarily be extrapolated to all LPVs.

By contrast, the situation observed in S Cam (Fig. 14) is quite interesting: although the CCF shape is almost identical for similar phases of two consecutive cycles, it is shifted by about $3 \mathrm{~km} \mathrm{~s}^{-1}$. Figure 15 shows the radialvelocity curve of S Cam: the common velocity shift for the blue and red peaks from one cycle to the next is quite clear, and flags this star as a suspected binary. The example of S Cam only serves to illustrate the method, since to confirm the binary nature of S Cam would still require us to prove that the phases compared in Fig. 14 are really identical, i.e. that the period is constant (this is not necessarily the case for a SRa variable like S Cam) and that the observed radial-velocity shift is larger than the typical cycle-to-cycle scatter.

Little is known about the binary frequency among LPVs (see Jorissen 2001 for a recent review). As pointed out in Sect. 4.1 and Fig. 3, the radial-velocity variations induced by the LPV pulsation are large $\left(\Delta V_{\mathrm{r}} \sim 20 \mathrm{~km} \mathrm{~s}^{-1}\right.$ over $\sim 1 \mathrm{y}$ ) as compared to the variations expected for the orbital motion of a large giant in a relatively wide binary system $\left(\Delta V_{\mathrm{r}} \sim 5 \mathrm{~km} \mathrm{~s}^{-1}\right.$ over several years). 


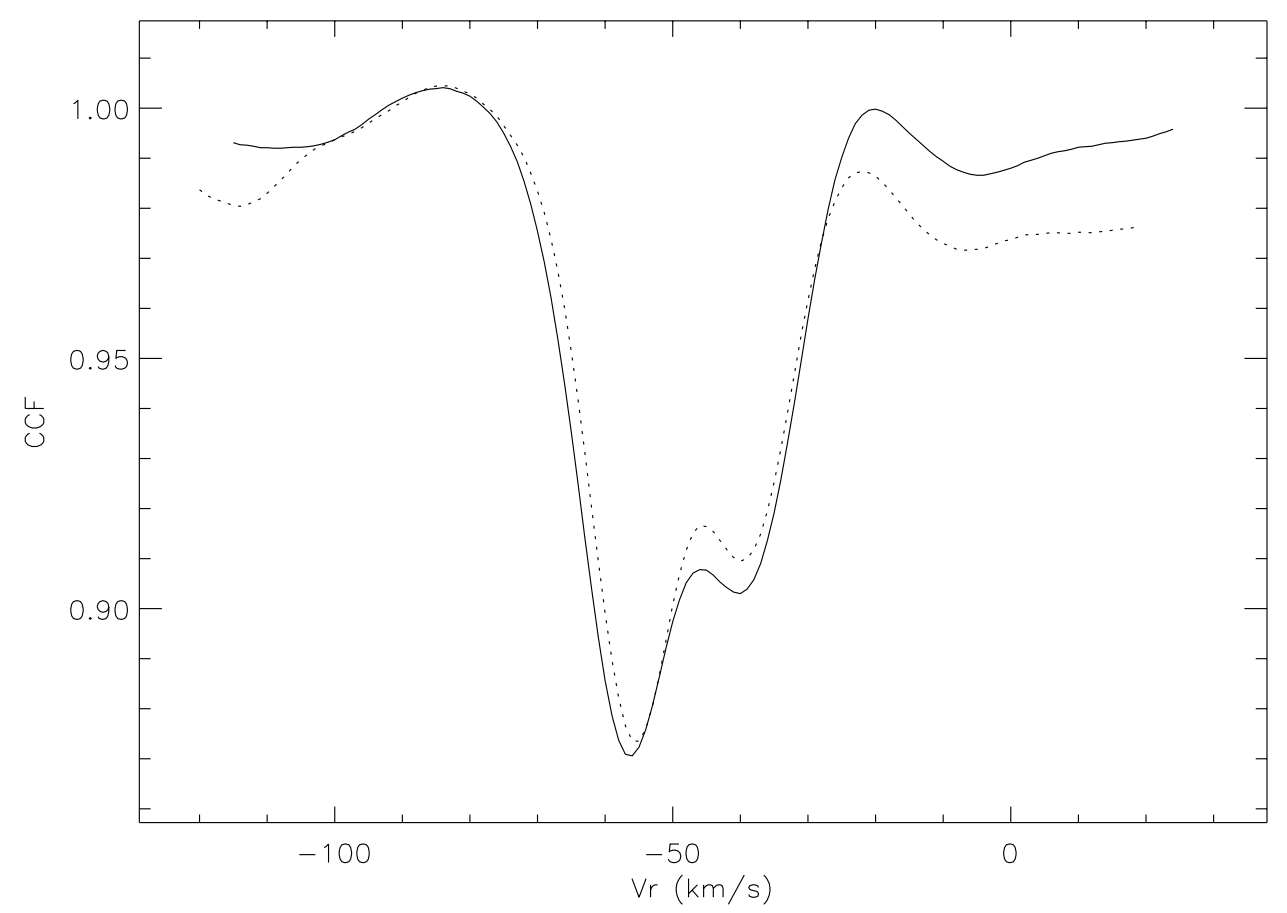

Fig. 12. Cross-correlation profiles of R Gem obtained with the default K0 III mask at phases 0.99 (solid line) and 2.09 (dotted line). Velocities are $\left(V_{\text {blue }}, V_{\text {red }}\right)=(-56.6,-38.8) \mathrm{km} \mathrm{s}^{-1}$ (phase 0.99) and $(-55.8,-39.2) \mathrm{km} \mathrm{s}^{-1}$ (phase 2.09).

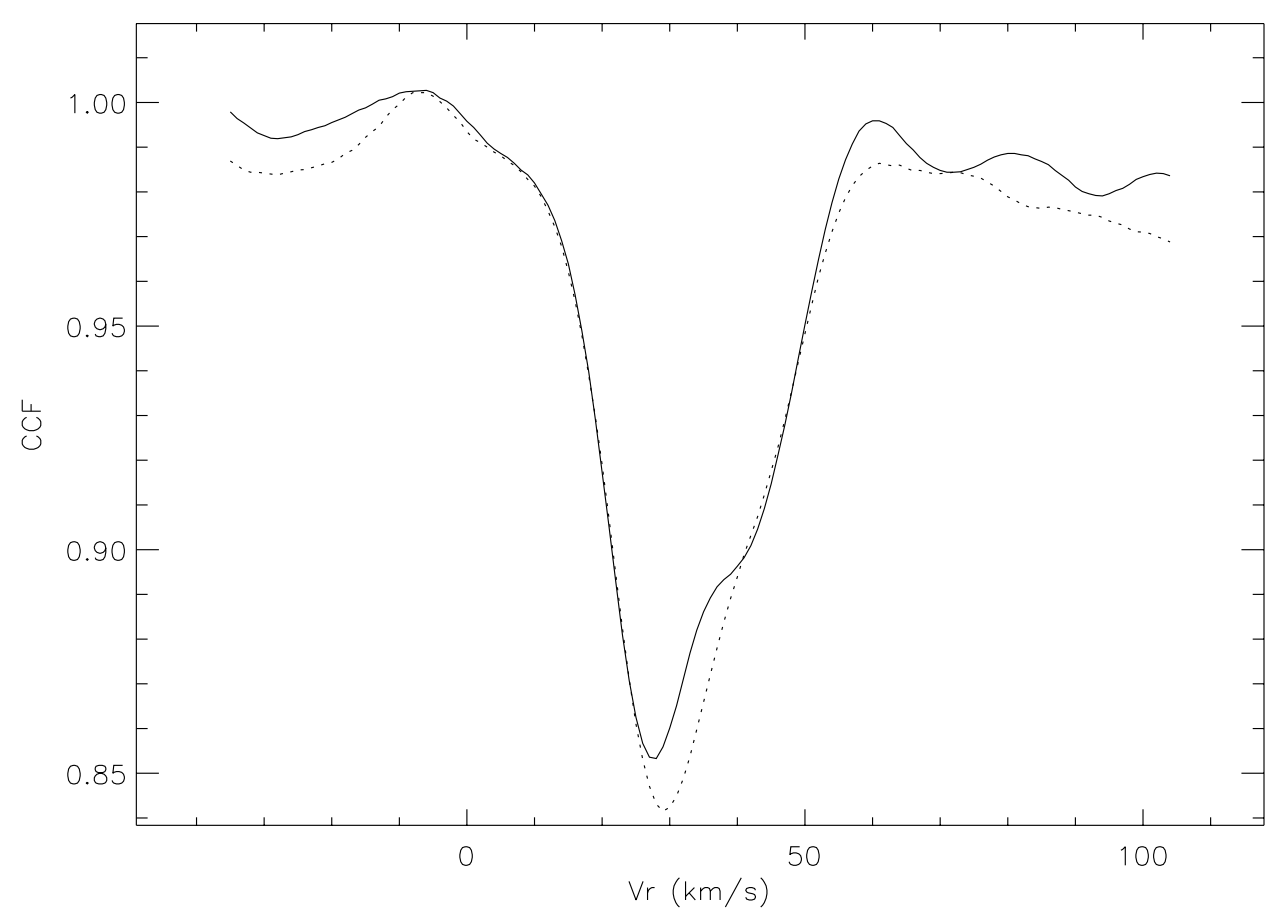

Fig. 13. Cross-correlation profiles of ST And obtained with the default K0 III mask at phases 0.99 (solid line) and 1.98 (dotted line). Velocities are $\left(V_{\text {blue }}, V_{\text {red }}\right)=(+27.5,+43.1) \mathrm{km} \mathrm{s}^{-1}$ (phase 0.99) and $(+28.7,+43.5) \mathrm{km} \mathrm{s}^{-1}$ (phase 1.98).

However, the example of SCam provides a hint on how binary systems may be found among LPVs exhibiting a double-peak CCF: a similar velocity shift observed for both the red and blue components from one cycle to the other may be indicative of a binary motion. Such a strategy is unfortunately quite time-consuming as only double-peak CCFs obtained around the same pulsational phase in successive cycles may be used, and is obviously not applicable to those LPVs exhibiting only a single-peak CCF.

In our sample of $81 \mathrm{LPVs}, 43$ showed a doublepeak CCF at least once with the K0-template (Table 3), but only 9 could be observed at about the same phase (within 0.1) in consecutive cycles and exhibited 


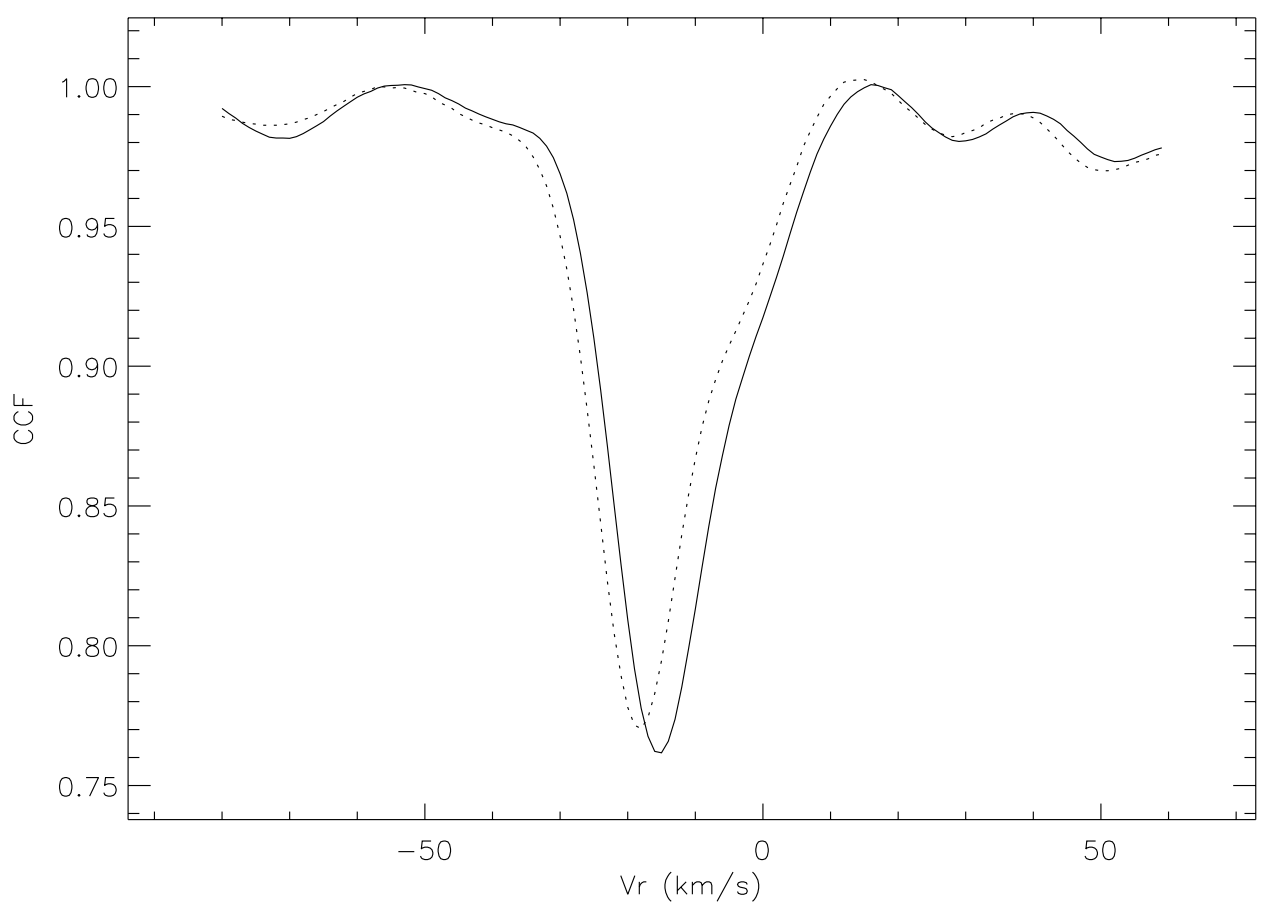

Fig. 14. Cross-correlation profiles of S Cam obtained with the default K0 III mask at phases 0.15 (solid line) and 1.13 (dotted line). Velocities are $\left(V_{\text {blue }}, V_{\text {red }}\right)=(-15.4,-0.9) \mathrm{km} \mathrm{s}^{-1}$ (phase 0.15) and $(-18.2,-3.1) \mathrm{km} \mathrm{s}^{-1}$ (phase 1.13).

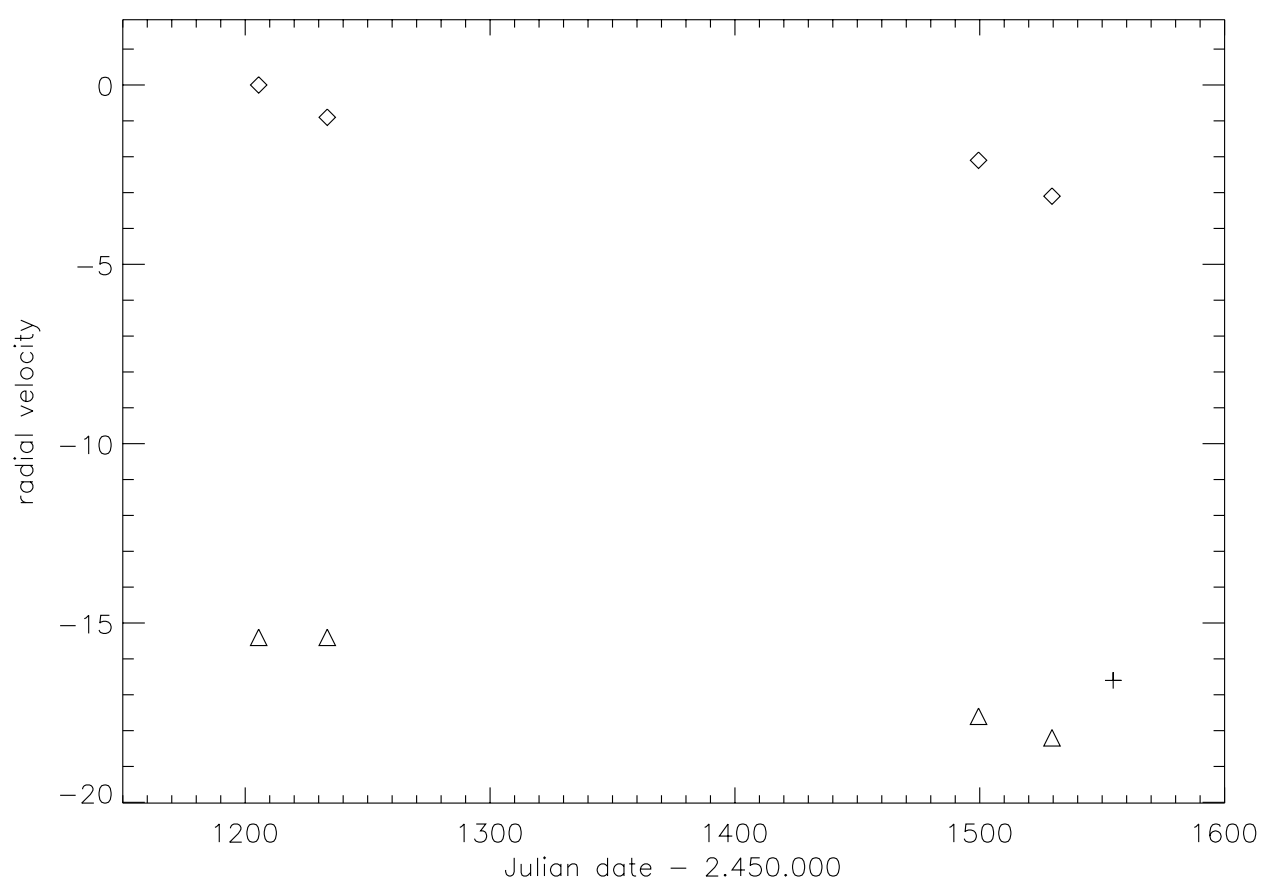

Fig. 15. Radial-velocity curve of S Cam with the default K0 III mask. Symbols are as in the last panel of Fig. 3. Notice the long-term trend, unlike the situation observed for S Cep in Fig. 3.

double-peak CCFs (namely Y And, V Tau, S Cam, R Gem, Y Vir, Z Oph, RT Cyg, S Cep and ST And). In this sample, the average absolute velocity shift between similar phases in consecutive cycles amounts to $\overline{\left|\Delta V_{\text {blue }}\right|}=1.3 \pm 0.6$ (rms) $\mathrm{km} \mathrm{s}^{-1}$ and $\overline{\left|\Delta V_{\text {red }}\right|}=1.4 \pm 1.1(\mathrm{rms}) \mathrm{km} \mathrm{s}^{-1}$ when removing the suspected binaries SCam (having $\left|\Delta V_{\text {blue }}\right|=2.2$ and $2.8 \mathrm{~km} \mathrm{~s}^{-1}$, and $\left|\Delta V_{\text {red }}\right|=2.1$ and $2.2 \mathrm{~km} \mathrm{~s}^{-1}$ ) and, somewhat less likely, Y Vir (having
$\left|\Delta V_{\text {blue }}\right|=3.0 \mathrm{~km} \mathrm{~s}^{-1}$, and $\left.\left|\Delta V_{\text {red }}\right|=2.2 \mathrm{~km} \mathrm{~s}^{-1}\right)$. No other star in the above list shows a significant signature of binary motion.

For the stars not suspected of being binaries, the red- and blue-peak shifts from one cycle to the next may sometimes be quite different, since the average $\left|\Delta V_{\text {blue }}-\Delta V_{\text {red }}\right|$, instead of being close to 0 , amounts to $1.8 \pm 1.5$ (rms) $\mathrm{km} \mathrm{s}^{-1}$. 


\section{Summary}

The most striking result relative to the statistics of occurrence of the line-doubling phenomenon in a sample of 81 LPVs of various periods, spectral types and brightness ranges is the fact that the compact LPVs are more prone to exhibit the line-doubling phenomenon than largesize LPVs (at least when comparing their K0III CCFs). The possibility that this segregation is an artefact of the use of the K0III template may not be totally excluded, since warmer masks (F0V and G2V) applied to the most extended and coolest LPVs yield asymmetric cross-correlation functions. This suggests that line doubling is occurring in those stars as well. Although a firm conclusion on this point is hampered by the large correlation noise present in the CCFs of cool LPVs obtained with warm masks, the occurrence of line doubling in those stars is confirmed by the double CO $\Delta v=3$ lines observed around $1.6 \mu \mathrm{m}$ by Hinkle et al. (1984). Moreover, the $\mathrm{H} \delta$ line in emission, which is another signature of the presence of shocks, is observed as well in the most extended stars, although with a somewhat narrower profile. This is an indication that the shock is weaker in extended than in compact LPVs, which may also contribute to the difficulty of detecting line doubling in cool, extended LPVs.

Stars with double absorption lines around maximun light exhibit S-shaped radial-velocity curves similar to the one observed for infrared lines.

A comparison with the center-of-mass (COM) velocity obtained from submm CO lines originating in the circumstellar envelope reveals that the median velocity between the red and blue peaks is blueshifted with respect to the COM velocity, as expected if the shock moves upwards.

Acknowledgements. RA benefits of a TMR "Marie Curie" Fellowship at ULB. A.J. is Research Associate from the Fonds National de la Recherche Scientifique (Belgium). We thank J. Mattei for providing data from the AAVSO database. This program would not have been possible without the generous allocation of telescope time at the Observatoire de Haute-Provence (operated by CNRS, France). We tank S. Udry for providing us with F0V and G2V templates.

\section{References}

Adams, W. S. 1941, ApJ, 93, 11

Alvarez, R., Jorissen, A., Plez, B., Gillet, D., \& Fokin, A. 2000a, A\&A, 362, 655, Paper I

Alvarez, R., Lançon, A., Plez, B., \& Wood, P. R. 2000b, A\&A, 353,322

Alvarez, R., Jorissen, A., Plez, B., et al. 2001, A\&A, 379, 288, Paper II

Baranne, A., Mayor, M., \& Poncet, J. L. 1979, Vistas Astron., 23, 279

Baranne, A., Queloz, D., Mayor, M., et al. 1996, A\&AS, 119, 373

Barnbaum, C. 1992, AJ, 104, 1585

Bessell, M. S., Scholz, M., \& Wood, P. R. 1996, A\&A, 307, 481
Bessell, M. S., Castelli, F., \& Plez, B. 1998, A\&A, 333, 231

Chadid, M., \& Gillet, D. 1996, A\&A, 308, 481

Delfosse, X., Forveille, T., Beuzit, J.-L., et al. 1999, A\&A, 344, 897

Deutsch, A. J., \& Merrill, P. W. 1959, ApJ, 130, 570

Fadeyev, Yu. A., \& Gillet, D. 2000, A\&A, 354, 349

Fadeyev, Yu. A., \& Gillet, D. 2001, A\&A, in press

Feast, M. W. 1963, MNRAS, 125, 367

Ferlet, R., \& Gillet, D. 1984, A\&A, 133, L1

Feast, M. W., Glass, I. S., Whitelock, P. A., \& Catchpole, R. M. 1989, MNRAS, 241, 375

Fox, M. W., \& Wood, P. R. 1985, ApJ, 297, 455

Fox, M. W., Wood, P. R., \& Dopita, M. A. 1984, ApJ, 286, 337

Fujita, Y. 1951, ApJ, 113, 626

Gillet, D. 1988a, A\&A, 190, 200

Gillet, D. 1988b, A\&A, 192, 206

Gillet, D., \& Lafon, J.-P. 1983, A\&A, 128, 53

Gillet, D., \& Lafon, J.-P. 1984, A\&A, 139, 401

Gillet, D., \& Lafon, J.-P. 1990, A\&A, 235, 255

Gillet, D., Maurice, E., \& Baade, D. 1983, A\&A, 128, 384

Gillet, D., Maurice, E., Bouchet, P., \& Ferlet, R. 1985a, A\&A, 148, 155

Gillet, D., Ferlet, R., Maurice, E., \& Bouchet, P. 1985b, A\&A, 150, 89

Gillet, D., Lafon, J.-P., \& David, P. 1989, A\&A, 220, 185

Gorbatskii, V. G. 1957, SvA, 1, 834

Gorbatskii, V. G. 1961, SvA, 5, 192

Groenewegen, M. A. T., Baas, F., Blommaert, J. A. D. L., et al. 1999, A\&AS, 140, 197

Habing, H. 1996, ARA\&A, 7, 97

Houdashelt, M. L., Bell, R. A., Sweigart, A. V., \& Wing, R. F. 2000, AJ, 119, 1424

Hill, S. J., \& Willson, L. A. 1979, ApJ, 229, 1029

Hinkle, K. H. 1978, ApJ, 220, 210

Hinkle, K. H., \& Barnbaum, C. 1996, AJ, 111, 913

Hinkle, K. H., \& Barnes, T. G. 1979a, ApJ, 227, 923

Hinkle, K. H., \& Barnes, T. G. 1979b, ApJ, 234, 548

Hinkle, K. H., Hall, D. N. B., \& Ridgway, S. T. 1982, ApJ, 252, 697

Hinkle, K. H., Scharlach, W. W. G., \& Hall, D. N. B. 1984, ApJS, 56, 1

Hinkle, K. H., Lebzelter, T., \& Scharlach, W. W. G. 1997, AJ, 114,2686

Hofmann, K-H., Balega, Y., Scholz, M., \& Weigelt, G. 2000, A\&A, 353, 1016

Jorissen, A. 2001, in Asymptotic Giant Branch Stars, ed. H. Habing, \& H. Olofsson (Springer Verlag, Berlin), in press

Joy, A. H. 1947, ApJ, 106, 288

Joy, A. H. 1954, ApJS, 1, 39

Karp, A. H. 1975, ApJ, 201, 641

Kholopov, P. N., Samus, N. N., Frolov, M. S., et al. 1988, General Catalogue of Variable Stars, Fourth Edition (Nauka Publ. House, Moscow)

Knapp, G. R., Young, K., Lee, E., \& Jorissen, A. 1998, ApJS, 117, 209

Lançon, A. 1999, in AGB Stars, ed. T. Le Bertre, A. Lèbre, \& C. Waelkens, IAU Symp., 191, ASP Conf. Ser., 579

Maehara, H. 1968, PASJ, 20, 77

Maehara, H. 1971, PASJ, 23, 503

Magnan, C., \& de Laverny, P. 1997, MNRAS, 286, 920

Mattei, J. A. 2000, Observations from the AAVSO International Database, private communication 
Merrill, P. W. 1921, ApJ, 53, 185

Merrill, P. W. 1923, ApJ, 58, 215

Merrill, P. W. 1947, ApJ, 105, 360

Merrill, P. W. 1955, PASP, 67, 199

Merrill, P. W., \& Burwell, C. G. 1930, ApJ, 71, 285

Merrill, P. W., \& Greenstein, J. L. 1958, PASP, 70, 98

Neri, R., Kahane, C., Lucas, R., Bujarrabal, V., \& Loup, C 1998, A\&AS, 130, 1

Parsons, S. B. 1972, ApJ, 174, 57

Perrin, G., Coudé du Foresto, V., Ridgway, S. T., et al. 1998, A\&A, 331, 619

Perrin, G., Coudé du Foresto, V., Ridgway, S. T., et al. 1999, A\&A, 345, 221

Queloz, D. 1995, Echelle spectroscopy with a CCD at low signal-to-noise ratio, in New developments in array technology and applications, IAU Symp. 167, ed. A. G. Davis Philip, K. A. Janes, \& A. R. Upgren (Kluwer, Dordrecht), 221 de la Reza, R. 1986, in Quasi-thermal models, ed. H. R. Johnson, \& F. R. Querci, The M-type stars (NASA SP492), NASA and CNRS, 373

Schwarzschild, M. 1952, in Shock waves in the atmosphere of pulsating stars, ed. P. T. Oosterhoff, Transactions of the IAU, vol. VIII (Cambridge University Press), 811

Serote-Roos, M., Boisson, C., \& Joly, M. 1996, A\&AS, 117, 93 Tsuji, T. 1971, PASJ, 23, 275

Wallerstein, G. 1985, PASP, 97, 994

Wallerstein, G., Hinkle, K. H., Dominy, J. F., et al. 1985, MNRAS, 215, 67

Willson, L. A. 1972, A\&A, 17, 354

Willson, L. A. 1976, ApJ, 205, 172

Willson, L. A., \& Hill, S. J. 1979, ApJ, 228, 854

Willson, L. A., Wallerstein, G., \& Pilachowski, C. A. 1982, MNRAS, 198, 483

Wood, P. R. 1979, ApJ, 227, 220 